\title{
Extraction of electromagnetic neutron form factors through inclusive and exclusive polarized electron scattering on polarized ${ }^{3}$ He target
}

\author{
J. Golak ${ }^{\dagger}$, G. Ziemer, H. Kamada*, H. Witała ${ }^{\dagger}$, W.Glöckle \\ Institut für Theoretische Physik II, Ruhr-Universität Bochum, D-44780 Bochum, Germany \\ $\dagger$ Institute of Physics, Jagellonian University, PL-30059 Cracow, Poland
}

(October 30, 2018)

\begin{abstract}
The inclusive and exclusive processes $\overrightarrow{{ }^{3} \mathrm{He}}\left(\vec{e}, e^{\prime}\right)$ and $\overrightarrow{{ }^{3} \mathrm{He}}\left(\vec{e}, e^{\prime} n\right)$ have been theoretically analyzed and values for the magnetic and electric neutron form factors have been extracted. In both cases the form factor values agree well with the ones extracted from processes on the deuteron. Our results are based on Faddeev solutions, modern NN forces and partially on the incorporation of mesonic exchange currents.
\end{abstract}

21.45.+v, 25.10.+s, 24.70.+s, 25.30.Fj

*present address: Institut für Strahlen- und Kernphysik der Universität Bonn Nussallee 14-16, D53115 Bonn, Germany 


\section{INTRODUCTION}

Besides the deuteron, polarized ${ }^{3} \mathrm{He}$ appears to be a useful target to extract information on the electromagnetic neutron form factors. This proposal goes back to [1] and has been emphasized again by [2]. It is based on the fact that the principal S-state dominates the ${ }^{3} \mathrm{He}$ wave function by more than $90 \%$ and in this state the polarization is carried solely by the neutron. Needless to say that the knowledge of the electromagnetic form factors of the neutron is basic to get insight into the distribution of charge and magnetization inside the neutron. We refer to [3-8] and [9] for experimental and theoretical work on that topic extracting information on the neutron form factors. In this article we would like to analyze two experiments [10,13] on the processes ${ }^{3} \mathrm{He}\left(\vec{e}, e^{\prime}\right)$ and $\overrightarrow{{ }^{3} \mathrm{He}}\left(\vec{e}, e^{\prime} n\right)$ with the aim to extract the magnetic and electric neutron form factors at certain $Q^{2}$ values. This theoretical analysis will be based on Faddeev solutions for the $3 \mathrm{~N}$ continuum and the $3 \mathrm{~N}$ bound state belonging to the same 3N Hamiltonian. We shall also use realistic $\mathrm{NN}$ forces. For the inclusive process we carried through an analysis before [14, but now it refers to a new more accurate experiment [10] and also the theory will be improved by including mesonic exchange currents. The analysis [15] of the exclusive experiment by consistent Faddeev solutions for the $3 \mathrm{~N}$ continuum and ${ }^{3} \mathrm{He}$ has not been done before to the best of our knowledge.

In section \I we shall investigate the inclusive process and in section III the exclusive one. We close with an outlook in section IV.

\section{EXTRACTION OF THE MAGNETIC FORM FACTOR OF THE NEUTRON}

In this section we shall analyze a measurement of $\overrightarrow{{ }^{3} \mathrm{He}}\left(\vec{e}, e^{\prime}\right)$ carried through at Jlab 10 . We refer to [14 for the detailed theoretical formalism and restrict ourselves to describe only its extensions. That article will henceforth be cited as I and equations thereof by (I.*). In I we used only a single nucleon current operator. Now we add two-body exchange current operators. The central Faddeev-like equation given in Eq. (I.28) is derived under the assumption that the operator $C$ has the form of (I.29). In I this simply meant that $C$ is a sum of three single particle operators. However, what really enters the derivation of Eq. (I.28) is, that the operator $C$ can be decomposed into three parts such that $C^{(i)}$ is symmetrical under exchange of particles $j \neq k$ with $j \neq i \neq k$. The operator $C$ has the physical meaning of a component of the current operator. Thus we can simply add two-body currents, which naturally decompose in a $3 \mathrm{~N}$ system into three parts and therefore $C^{(1)}$ in Eq. (I.28) will be now a sum of two terms:

$$
C^{(1)}=C_{\text {sing }}^{(1)}+C_{\text {exch }}^{(23)}
$$

The first term is the single-nucleon current used in I and $C_{\text {exch }}^{(23)}$ is the corresponding component of a two-body current acting on particles 2 and 3. As a consequence there will occur now an additional driving term in Eq. (I.28) of the form $\left(1+t G_{0}\right) C_{\mathrm{exch}}^{(23)} \mid \Psi_{3} \mathrm{He}_{e} m$.

The following steps in I concern the partial wave representation. Since the spherical

components $C_{ \pm 1}^{(23)}$ of the two-body current operator are tensor operators and behave like the single nucleon components used in I, the conditions (I.36-37) and as a consequence (I.38) remain valid. The symmetry properties (I.41-44) based on the partial wave decomposed 
forms remain also valid for the additional two-body currents. This follows from their explicit forms as given in [16]. Then the following expressions leading to the final forms of the four response functions (I.52-55) remain valid.

For the two-body currents we follow the Riska prescription [17], which via the continuity equation relates $\mathrm{NN}$ forces and exchange currents in a model independent manner, as it is often referred to. We choose the AV18 NN force model [18] and restrict ourselves to the dominant $\pi$ - and $\rho$-like parts. We refer to [19] for more details and to [16] for the partial wave expansion of the two-body currents. In case of Bonn B [20], which we also use as another NN force model, we choose standard $\pi$ - and $\rho$-meson exchange currents augmented by the strong form factors used in Bonn B. For the proton electromagnetic form factors we took the Höhler [25] parametrization, which at $Q^{2}=0.1$ and $0.2 \mathrm{GeV}^{2} / c^{2}$ agrees perfectly with the data.

To theoretically analyze the data from [10] the experimental conditions have to be taken into account. The incoming electron beam energy was $E=778 \mathrm{MeV}$. The central electron scattering angles for $Q^{2}=0.1$ and $0.2 \mathrm{GeV}^{2} / c^{2}$ were $\theta_{e}=24.44^{\circ}$ and $35.5^{\circ}$, respectively. The spread in the electron angles were $\Delta \theta_{e}= \pm 1.6^{\circ}$ and $\Delta \phi_{e}= \pm 3.4^{\circ}$. For $Q^{2}=0.1(0.2)$ $\mathrm{GeV}^{2} / c^{2}$ or values close to it the virtual photon energies were chosen between $30-90 \mathrm{MeV}$ $(80-140 \mathrm{MeV})$ with a central value of $60 \mathrm{MeV}(110 \mathrm{MeV})$. Each of these two $\omega$-ranges were divided into 7 bins of length $10 \mathrm{MeV}$.

The three-fold cross section for inclusive scattering has the well known form (Eq. (I.3))

$$
\frac{d^{3} \sigma}{d \hat{k}^{\prime} d k_{0}^{\prime}}=\sigma_{\mathrm{Mott}}\left\{v_{L} R^{L}+v_{T} R^{T}+h\left(v_{T L^{\prime}} R^{T L^{\prime}}+v_{T^{\prime}} R^{T^{\prime}}\right)\right\} .
$$

Here $\sigma_{\text {Mott }}$ is the Mott cross section, $v_{i}$ are analytically known kinematical factors and the $R^{i}$ are inclusive response functions divided into two groups. The first one is present for unpolarized electrons, the second one goes with the helicity $h$ of the electron beam. The primed response functions also depend on the orientation of the ${ }^{3} \mathrm{He}$ spin in relation to the photon direction (see Eqs. (I.56-57)). The corresponding angles are denoted by $\theta^{\star}$ and $\phi^{\star}$. The cross section in Eq. (2) was averaged over the $10 \mathrm{MeV}$ wide $\omega$-bins and over the angular spread around the central electron scattering angles. In order to perform the averaging a sufficiently fine grid in $\theta_{e}$ and $\omega$ has been chosen for which the four response functions have been calculated. This required quite a few hundred solutions of the corresponding Faddeev equations. The actual averaging was performed via a Monte Carlo procedure based on the response functions known on the grid of electron angles and electron energies. This Monte Carlo procedure takes into account the finite momentum and angular acceptance of the experiment.

The asymmetry is defined as

$$
A=\frac{\left.\frac{d^{3} \sigma}{d \hat{k}^{\prime} d k_{0}^{\prime}}\right|_{h=1}-\left.\frac{d^{3} \sigma}{d \hat{k}^{\prime} d k_{0}^{\prime}}\right|_{h=-1}}{\left.\frac{d^{3} \sigma}{d \hat{k}^{\prime} d k_{0}^{\prime}}\right|_{h=1}+\left.\frac{d^{3} \sigma}{d \hat{k}^{\prime} d k_{0}^{\prime}}\right|_{h=-1} .}
$$

This ratio is formed out of the averaged cross sections. As a consequence one arrives at (see Eq. (I.58)) 


$$
A_{\text {average }}=\frac{\int d \Omega \sigma_{\mathrm{Mott}}\left\{v_{T^{\prime}} \tilde{R}^{T^{\prime}} \cos \theta^{\star}+v_{T L^{\prime}} \tilde{R}^{T L^{\prime}} \sin \theta^{\star} \cos \phi^{\star}\right\}}{\int d \Omega \sigma_{\mathrm{Mott}}\left\{v_{L} R^{L}+v_{T} R^{T}\right\}} \equiv \frac{\Delta}{\Sigma},
$$

where $d \Omega$ stands for the averaging. (We factored off the $\theta^{\star} \phi^{\star}$-dependence introducing the response functions with tilde). For $\theta^{\star}=0^{\circ}$ or close to it one focuses on $\tilde{R}^{T^{\prime}}$ and a corresponding $A_{T^{\prime}}$, which in a plane wave impulse approximation (PWIA) is essentially proportional to $\left(G_{M}^{n}\right)^{2}$ (see Eq. (I.77)). In the actual experiment one has to live with $\theta^{\star} \leq 10^{\circ}\left(7.8^{\circ}\right)$ for $Q^{2}=0.1(0.2) \mathrm{GeV}^{2} / c^{2}$ and the corresponding $\phi^{\star}$ is close to $0^{\circ}$ or $180^{\circ}$.

The searched for magnetic form factor of the neutron was parameterized as

$$
\left.G_{M}^{n}\left(Q^{2}\right) \equiv \lambda G_{M}^{n}\left(Q^{2}\right)\right|_{\text {model }},
$$

where $\left.G_{M}^{n}\left(Q^{2}\right)\right|_{\text {model }}$ was taken from [25]. In order to keep the computer time below an acceptable limit the averaging process was performed only for $\lambda=1$. For the $\lambda$-values in the neighborhood of 1 it was assumed that the change for $A_{T^{\prime}}$ from point geometry (fixed $\omega$ and central electron angles) to the averaged case is the same as for $\lambda=1$. Because of the smallness of the $\lambda$ interval around $\lambda=1$ (see below) this is highly plausible. In this manner one generated for each $\lambda$-value theoretical $A_{T^{\prime}}$-values according to the seven $\omega$-bins. The final step is the adjustment of the magnetic form factor of the neutron, $G_{M}^{n}$. Out of the seven $\omega$-bins three central values in the QFS region were selected (for $Q^{2}=0.1 \mathrm{GeV}^{2} / c^{2}$ $\omega=50,60,70 \mathrm{MeV}$ and for $Q^{2}=0.2 \mathrm{GeV}^{2} / c^{2} \omega=100,110,120 \mathrm{MeV}$ ) and an additional averaging was performed

$$
\bar{A} \equiv \frac{\sum_{j=1}^{3} A_{j} \Sigma_{j}}{\sum_{i=1}^{3} \Sigma_{i}}=\sum_{j=1}^{3} \frac{\Delta_{j}}{\Sigma_{j}} \frac{\Sigma_{j}}{\sum_{i=1}^{3} \Sigma_{i}} \equiv \sum_{j=1}^{3} \frac{\Delta_{j}}{\Sigma_{j}} w_{j}=\sum_{j=1}^{3} A_{j} w_{j}
$$

The indices $i$ and $j$ refer to the three experimental bins. In the third equality weight factors $w_{j}$ are introduced, which in the actual performance were taken from the experiment (counts related to the unpolarized cross section).

In this manner one arrives at the $\lambda^{2}$-dependence of $\bar{A}$, which turned out to be rather close to a straight line. This is depicted in Figs. 1 and a for $Q^{2}=0.1$ and $0.2 \mathrm{GeV}^{2} / c^{2}$, respectively, together with the experimental values. One reads off the $\lambda$-values leading to $G_{M}^{n}$ values as given in Table @. There $G_{M}^{n}($ dipole $)=\mu_{n} /\left(1+Q^{2} / 0.71\right)^{2}$, where $\mu_{n}$ is the magnetic moment of the neutron.

Having adjusted $G_{M}^{n}$ we can display the $\omega$-dependence of $A_{T^{\prime}}$ in Figs. 3 and $甘$ in comparison to the experimental values. We see an essentially perfect agreement between theory and experiment. We also show the theoretical result without MEC's but including the full final state interaction. Clearly the MEC's provide an important shift and should be not neglected. Also the final state interaction itself plays a very important role since the PWIA result (see Figs. 5 and 6) is far off. Note our PWIA neglects all final state interactions.

The electric form factor of the neutron, $G_{E}^{n}$, is not yet very well known (see section III), but enters into our calculation. Its effect is totally negligible as can be seen in Figs. 7 and 8. There we compare $A_{T^{\prime}}$ (for point geometry) evaluated with $G_{E}^{n}$ according to [25] and putting it to zero.

For point geometry we also performed full fledged calculations based on the AV18 NN force [18] and the $\pi$ - and $\rho$-like exchange currents according to the Riska prescription [17]. Both calculations, for Bonn B and AV18 agree very well as shown in Figs. 9 and 10 . 
Finally we mention that we also included $\pi$ - and $\rho$-exchange currents with an intermediate $\Delta$ (in a static approximation). Their effects was very small and lead to an estimated change of $G_{M}^{n}$ by less than $2 \%$.

The data in Figs. $3-10$ are radiatively corrected. This thorough procedure was performed with the help of numerous full fledged Faddeev calculations and will be described in a separate article [26].

Our $G_{M}^{n}$ value extracted from that inclusive experiment on ${ }^{3}$ He agrees very well with results achieved in recent experiments on the deuteron [7,8]. This is shown in Fig. 11 together with other data.

We refrained from a theoretical analysis of data taken in the same experiment at higher $Q^{2}$-values [10], since one has to expect that relativity will play a non-negligible role. This is left to a future investigation.

\section{EXTRACTION OF THE ELECTRIC FORM FACTOR OF THE NEUTRON}

In this section we shall analyze a measurement of $\overrightarrow{{ }^{3} \mathrm{He}}\left(\vec{e}, e^{\prime} n\right)$ carried through at MAMI 13 with the aim to extract $G_{E}^{n}$. Our theoretical formalism has been described in [21]. Nevertheless to clearly shed light onto the reactions going on after the virtual photon has been absorbed we would like to lay out the multiple rescatterings and their summation into a Faddeev like integral equation. In the literature erroneously often just the very first few terms are taken into account. In a graphical representation the full photoninduced break-up process is an infinite sum of the type shown in Fig. 12. We assumed the absorption of the photon on a single nucleon. Obviously the diagrams can be generalized by photon absorption processes on two or three nucleons. This infinite sequence of processes has its algebraic counterparts

$$
\begin{aligned}
N & =(j(1)+j(2)+j(3))\left|\Psi_{3_{H e}}\right\rangle \\
& +\left(t_{12}+t_{23}+t_{31}\right) G_{0}(j(1)+j(2)+j(3))\left|\Psi_{3}{ }\right\rangle \\
& +\sum_{i=1}^{3} \sum_{k<l} \sum_{m<n \neq k<l} t_{m n} G_{0} t_{k l} G_{0} j(i)\left|\Psi_{3 H e}\right\rangle+\cdots
\end{aligned}
$$

It is convenient to introduce the notation $t_{i j} \equiv t_{k}(i j k=123$ etc. $)$ and $P \equiv P_{12} P_{23}+$ $P_{13} P_{23}$. Then it requires little work to put Eq. (7) into the form

$$
\begin{aligned}
N & =(1+P) j(1)\left|\Psi_{3_{H e}}\right\rangle \\
& +(1+P) t_{1} G_{0}(1+P) j(1)\left|\Psi_{3^{3} \mathrm{He}}\right\rangle \\
& +(1+P) t_{1} G_{0} P t_{1} G_{0}(1+P) j(1)\left|\Psi_{3_{H e}}\right\rangle+\cdots
\end{aligned}
$$

The first term contains no final state interaction and we split off, what we shall call plane wave impulse approximation (PWIA),

$$
N^{\mathrm{PWIA}} \equiv j(1)\left|\Psi_{3} \mathrm{He}_{e}\right\rangle
$$

and call the whole term

$$
N^{\text {PWIAS }} \equiv(1+P) j(1)\left|\Psi_{3}{ }_{H e}\right\rangle
$$


where S stands for full antisymmetrisation. All the additional terms contain rescattering contributions of increasing oder in the $\mathrm{NN} t$-operator:

$$
\begin{aligned}
N^{\text {rescatt }} & \equiv(1+P)\left[t_{1} G_{0}+t_{1} G_{0} P t_{1} G_{0}+\cdots\right](1+P) j(1)\left|\Psi_{3_{H e}}\right\rangle \\
& \equiv(1+P)|U\rangle .
\end{aligned}
$$

A more general inspection reveals that

$$
|U\rangle=\left(1+t_{1} G_{0} P+t_{1} G_{0} P t_{1} G_{0} P+\cdots\right) t_{1} G_{0}(1+P) j(1)\left|\Psi_{3 \mathrm{He}}\right\rangle,
$$

where inside the bracket the operator sequence $t_{1} G_{0} P$ occurs in increasing powers. As an immediate consequence one derives

$$
|U\rangle=t_{1} G_{0}(1+P) j(1)\left|\Psi_{3_{H e}}\right\rangle+t_{1} G_{0} P|U\rangle
$$

which is the central integral equation for the amplitude $|U\rangle$. Via Eq. (11) it provides the whole rescattering amplitude (up to the symmetrisation $(1+P)$ ). This integral equation is of the Faddeev type because of the typical Faddeev structure of its kernel. The same kernel occurs for 3N scattering processes [22], only the driving term is different there.

An often used approximation for quasielastic processes in the literature is

$$
N \approx j(1)\left|\Psi_{3_{H e}}\right\rangle+t_{23} G_{0} j(1)\left|\Psi_{3_{H e}}\right\rangle \equiv N^{\mathrm{FSI} 23} .
$$

Here antisymmetrisation in the final state is neglected and one rescattering in the NN $t$ operator $t_{23}$ is only allowed for the two spectator nucleons (which do not absorb the photon). This amplitude is also sometimes called PWIA, but not in this article.

Needless to say that the full amplitude, now supplemented by the proper vector indices for the current operator is identical to the standard form of the nuclear matrix element

$$
N^{\mu}=\left\langle\Psi_{f}^{(-)}\left|\sum_{i} j^{\mu}(i)\right| \Psi_{3 H e}\right\rangle .
$$

We refer to [21] for the verification.

The sixfold differential cross section for the exclusive process under discussion has the well known form [23]

$$
\begin{gathered}
\frac{d^{6} \sigma}{d \hat{k}^{\prime} d k_{0}^{\prime} d \hat{p}_{n} d p_{n}}=\sigma_{\mathrm{Mott}} p_{n}^{2} \frac{p m_{N}}{2} \\
\times \int d \hat{p}\left\{v_{L} R^{L}+v_{T} R^{T}+v_{T T} R^{T T}+v_{T L} R^{T L}+h\left(v_{T L^{\prime}} R^{T L^{\prime}}+v_{T^{\prime}} R^{T^{\prime}}\right)\right\} .
\end{gathered}
$$

Here $\hat{k}^{\prime}, k_{0}^{\prime}, \hat{p}_{n}, p_{n}, p, \hat{p}$ in turn are the unit vector in the direction of the scattered electron, its energy, the unit vector of the knocked out neutron, its momentum, the magnitude of the relative momentum of the undetected two protons and finally the unit vector pointing into the direction of that relative momentum.

Throughout this article we use a strictly nonrelativistic notation.

The information on $G_{E}^{n}$ magnified by the product with $G_{M}^{n}$ is contained in $R_{T L^{\prime}}$ as can be explicitely seen working out PWIA [15] (see also [13]). In order to isolate the primed 
structure functions one forms an asymmetry $A$ of the cross section with respect to the electron helicities $h= \pm 1$. One finds the well known result

$$
A=\frac{\int d \hat{p}\left(v_{T L^{\prime}} R^{T L^{\prime}}+v_{T^{\prime}} R^{T^{\prime}}\right)}{\int d \hat{p}\left(v_{L} R^{L}+v_{T} R^{T}+v_{T T} R^{T T}+v_{T L} R^{T L}\right)}
$$

In the experiment two perpendicular polarization axis for the spin of ${ }^{3} \mathrm{He}$ have been chosen, $\vec{S}_{\|}$and $\vec{S}_{\perp}$, leading to $A_{\|}$and $A_{\perp}$. In an optimal set up $\vec{S}_{\|}$and $\vec{S}_{\perp}$ would be parallel and perpendicular to the direction of the virtual photon. Then it is well known from the expression for PWIA that under this approximation

$$
\frac{A_{\perp}}{A_{\|}} \propto \frac{G_{E}^{n}}{G_{M}^{n}}
$$

Therefore the aim will be to extract $G_{E}^{n}$ from the measured value of that ratio. This is under the assumption that $G_{M}^{n}$ is sufficiently well known. Our contribution in this article is to show that the final state interaction (FSI) does not wash out the signal for $G_{E}^{n}$ and that taking FSI into account is crucial.

We shall now describe the experimental conditions under which the data were taken. The electron- and neutron-detectors covered a wide range of angles as displayed in Figs. 13. 14. Depending on the electron scattering angle $\theta_{e}$ only neutron momenta within certain cuts were accepted as shown in Table II. Since the energy of the scattered electron was not measured (except for excluding pion-production) the direction of the virtual photon was not known. However it was possible to correlate the directions of the photon and the knocked out neutron in the following manner [11]. Take the relative momentum $p$ of the two undetected protons to be zero. Then for fixed values of $\hat{k}^{\prime}, \hat{p}_{n}$ and $p_{n}, k_{0}^{\prime}$ and therefore $\vec{Q}$, the virtual photon momentum, follow kinematically. Now only those angles were allowed such that the angle between $\vec{Q}$ and $\hat{p}_{n}$ was smaller or equal to $6^{\circ}$. In our theoretical analysis we also took that constraint into account. As an example we show in Fig. 15 the allowed region for the neutron angles for given values of $\theta_{e}=43^{\circ}, \phi_{e}=0^{\circ}, p_{n}=530 \mathrm{MeV} / \mathrm{c}$ and $p=0$ (solid curve). In reality there is a distribution of $p$-values, see Fig. 16 below. Consequently for that region of neutron angles also other events with $p \neq 0$ contribute, which belong to different directions of $\vec{Q}$. This is also shown in Fig. 15. In the worst case the angle between $\hat{Q}$ and $\hat{p}_{n}$ can be as large as $9^{\circ}$ for the tails of the $p$-distribution.

Since the energy of the scattered electron has not been measured the cross section reduces to a five-fold one

$$
\begin{gathered}
\frac{d^{5} \sigma}{d \hat{k}^{\prime} d \hat{p}_{n} d p_{n}}=\frac{m_{N}}{2} \sigma_{\text {Mott }} p_{n}^{2} \\
\times \int d k^{\prime}{ }_{0} p \int d \hat{p}\left\{v_{L} R^{L}+v_{T} R^{T}+v_{T T} R^{T T}+v_{T L} R^{T L}+h\left(v_{T L^{\prime}} R^{T L^{\prime}}+v_{T^{\prime}} R^{T^{\prime}}\right)\right\} .
\end{gathered}
$$

We convert the $k_{0}^{\prime}$ integration into one over $p$, take into account the experimental acceptances and end up with the summed up cross section

$$
\Delta \sigma \equiv \int_{\Delta \hat{k}^{\prime}} d \hat{k}^{\prime} \sigma_{\mathrm{Mott}} \int_{\Delta p_{n}} d p_{n} p_{n}^{2} \int_{\Delta \hat{p}_{n}} d \hat{p}_{n} \int d \vec{p} \rho
$$




$$
\begin{gathered}
\times\left\{v_{L} R^{L}+v_{T} R^{T}+v_{T T} R^{T T}+v_{T L} R^{T L}+h\left(v_{T L^{\prime}} R^{T L^{\prime}}+v_{T^{\prime}} R^{T^{\prime}}\right)\right\} . \\
\equiv \int d \Omega\left\{v_{L} R^{L}+v_{T} R^{T}+v_{T T} R^{T T}+v_{T L} R^{T L}+h\left(v_{T L^{\prime}} R^{T L^{\prime}}+v_{T^{\prime}} R^{T^{\prime}}\right)\right\} .
\end{gathered}
$$

In our nonrelativistic formulation $\rho$ has the form

$$
\rho=\frac{2 m_{N} p^{2}}{\sqrt{\left(k \cos \theta_{e}-\vec{p}_{n} \cdot \hat{k}^{\prime}-2 m_{N}\right)^{2}+4 m_{N}\left(k+\epsilon_{3 e}\right)-4 p^{2}-3 p_{n}^{2}-k^{2}+2 \vec{p}_{n} \cdot \vec{k}}}
$$

Since we shall form asymmetries there is no need to determine the value of the covered phase space. Using $\Delta \sigma$ for the two ${ }^{3} \mathrm{He}$-spin directions one can form the two asymmetries $A_{\perp}, A_{\|}$for the $\perp$ and $\|$ orientations of the ${ }^{3} \mathrm{He}$ spin and finally their ratio $V$

$$
V \equiv \frac{A_{\perp}}{A_{\|}}=\frac{\int d \Omega\left(v_{T L^{\prime}} R^{T L^{\prime}}+v_{T^{\prime}} R^{T^{\prime}}\right)_{\perp}}{\int d \Omega\left(v_{T L^{\prime}} R^{T L^{\prime}}+v_{T^{\prime}} R^{T^{\prime}}\right)_{\|}} \cdot 1
$$

The "1" in Eq. (22) denotes the corresponding ratio for the helicity independent parts of $\Delta \sigma$. It turned out that this latter ratio was extremely close to 1 (within less than $0.1 \%$ ).

Before we shall present our results for $V$ as a function of a parametrisation of $G_{E}^{n}$ we would like to give some insight into the functions entering Eq. (22). For some fixed directions of $\hat{k}^{\prime}$ and $\hat{p}_{n}$ and some value of $p_{n}$ contained in the domain $\Omega$ we define the quantities

$$
\Lambda_{\beta}^{\alpha} \equiv \int d \hat{p} R_{\beta}^{\alpha}
$$

which depend on $p$. For $\alpha$ we choose $T^{\prime}$ and $T L^{\prime}$ and $\beta$ corresponds to $\perp$ and $\|$-orientations of the ${ }^{3} \mathrm{He}$ spin. Fig. 16 tells us that indeed the distribution of the $p$-values is peaked at low values, where the maxima occur at kinetic energy values of relative motion of the two protons of about $0.4 \mathrm{MeV}$. At around $140 \mathrm{MeV} / \mathrm{c}$ the $p$-distribution has essentially vanished. Those curves in Fig. 16 refer to full FSI. In contrast the corresponding curves for PWIA, displayed in Fig. 17, show a much wider $p$-distribution, which has intriguing consequences as described below.

Next let us choose a fixed $k_{0}^{\prime}$-value, $k_{0}^{\prime}=650 \mathrm{MeV} / \mathrm{c}$, and again fixed angles $\theta_{e}=40^{\circ}$, $\phi_{e}=0^{\circ}, \theta_{n}=49.48^{\circ}$, and $\phi_{n}=180^{\circ}$ all chosen out of the large domain $\Omega$. In Fig. 18 we display the magnitudes of one of the amplitudes $\Lambda_{\perp}^{T^{\prime}}$, now as a function of $p_{n}$. The others are qualitatively similar. We compare different approximate treatments of the final state to the full calculation. The pure PWIA drops strongly with decreasing $p_{n}$. This is a simple consequence of the fact that the ${ }^{3} \mathrm{He}$ wave function drops with increasing momenta. Choosing Jacobi momenta as arguments of the ${ }^{3} \mathrm{He}$ wave function the photon momentum $\vec{Q}$ enters as $\vec{q}=\vec{p}_{n}-\vec{Q}$, where $\vec{q}$ is the relative momentum of the neutron in relation to the two protons. The decrease of the ${ }^{3} \mathrm{He}$ wave function with increasing $q$ explains the PWIA curve in Fig. 18. In case of the symmetrized PWIAS the photon can also be absorbed by the two protons, which leads to $\vec{q}=\vec{p}_{n}$ and the occurrence of $\vec{Q}$ in the other Jacobi momentum $\vec{p}_{23}$ as $\vec{p}_{23}=\vec{p} \mp \frac{1}{2} \vec{Q}$. As a consequence the two additional amplitudes in PWIAS start to contribute at lower $p_{n}$-values, which can clearly be seen in Fig. 18. The curve denoted as 
FSI23 is based on the final state interaction among the two final protons. This reduced final state interaction has apparently a strong effect even near the quasi elastic peak. Again including full antisymmetrisation in the final state, but keeping only a first order final state interaction, leads to strong deviations at low neutron momenta. This is denoted by FSI23S in Figs. 18-22. Finally the full FSI (including of course antisymmetrisation in the final state) leads to a behavior, which is similar to FSI23 near the upper end of $p_{n}$ but deviates then from all other curves for lower $p_{n}$ values.

Since the experiment under discussion emphasizes the large $p_{n}$-values in accordance with at least approximate quasi-free scattering conditions, we display in Figs. 19-22 the magnitudes of the four $\Lambda_{\beta}^{\alpha}$ amplitudes restricted to the domain seen in the experiment. We see a coincidence of PWIA and PWIAS in the restricted $p_{n}$ interval and a spread of curves for the other cases. Especially the FSI is clearly distinct from FSI23 for $R_{\|}^{T L^{\prime}}$ and $R_{\perp}^{T L^{\prime}}$.

The ratios of asymmetries for point geometries inside the domain $\Omega$ vary very much and depend extremely strongly on the treatment of the final $3 \mathrm{~N}$ state. A few more or less arbitrarily chosen cases are displayed in Figs. 23-25. In each case we see the ratio for PWIA, PWIAS, FSI23, FSI and an additional case, FSIn. In the latter case we put the electric form factor of the proton, $G_{E}^{p}$, to zero (the contribution of $G_{M}^{p}$ is insignificant [15]). This has been done to demonstrate the presence and importance of the photon absorption on the two protons. Consequently averaging over asymmetries related to point geometries is not advisable. Instead summing up cross sections first as in Eq. (20) and then forming asymmetries is what has to be done.

Let us now show our results for the ratio of asymmetries given in Eq. (22). We parametrize $G_{E}^{n}$ by multiplying three models for $G_{E}^{n}$ by a factor $\lambda$. We choose the ones by Gari-Krümpelmann [24]. Fig. 26 shows various theoretical ratios $V$ against $\lambda$ in comparison to the experimental value of $V^{\exp }=(-7.26 \pm 1.14) \%$ [12, 13]. The largest $\lambda$-value results for FSI, followed by PWIAS, then PWIA and finally FSI23. The four results for $\left.G_{E}^{n} \equiv \lambda \cdot G_{E}^{n}\right|_{\text {model }}$ are plotted in Fig. 27 in the range of $Q^{2}$ values touched in that experiment. This refers to one of the three models. Finally we show in Fig. $28 G_{E}^{n}$ as extracted through FSI and including the spread caused by the experimental error. Superimposed on the spread caused by the experimental error we see small variations due to the three different choices of $G_{E}^{n}$-models. For the central values around $Q^{2}=0.40 \mathrm{GeV}^{2} / c^{2}$ that model dependence is totally negligible. All our results are displayed in Table III], where we have taken the average of the highest and lowest values in Fig. 28. This average thus takes into account uncertainties of both, model dependence for $G_{E}^{n}$ and experimental errors.

It is astonishing that PWIAS (and PWIA) are relatively close to the value based on FSI. This is due to an accidental conspiracy. The slower decrease in the $p$-distribution for PWIA(S) shown in Fig. 17 causes smaller energies of the scattered electrons than for FSI. As a consequence the photon-direction deviates more strongly in case of $\vec{S}_{\perp}$ from $90^{\circ}$ than for FSI. This leads to a strongly modified contribution $\left(\int d \Omega v_{T^{\prime}} R^{T^{\prime}}\right)_{\perp}$ for PWIAS in comparison to using FSI. In addition because of the lacking FSI there are smaller protonic contributions. This together, as a detailed investigation shows [15], yields the accidental result, that PWIAS is close to the full result. The fact that PWIA(S) yields an unrealistic result could be verified by measuring the $p$-distribution for the response functions.

Our final result for FSI, $G_{E}^{n}=0.052 \pm 0.0038$ at $Q^{2}=0.40 \mathrm{GeV}^{2} / c^{2}$, is added in Fig. 29 to the ones extracted from processes on the deuteron. There is a fair agreement. Also added 
is another result achieved at MAMI [4] at a higher $Q^{2}$-value. No FSI corrections have been taken into account in that case.

Our present result leaves room for improvement. The effect of MEC's like in section II is still to be explored and due to the relatively high $Q^{2}$-value one cannot exclude that relativistic effects might be noticeable. This is left to future investigations.

\section{SUMMARY AND OUTLOOK}

We have extracted from two measurements on $\overrightarrow{{ }^{3} \mathrm{He}}\left(\vec{e}, e^{\prime}\right)$ and $\overrightarrow{{ }^{3} \mathrm{He}}\left(\vec{e}, e^{\prime} n\right)$ the magnetic and electric neutron form factors at certain $Q^{2}$-values. Our results are based on consistent Faddeev solutions for the $3 \mathrm{~N}$ continuum and the $3 \mathrm{~N}$ bound state. Modern $\mathrm{NN}$ forces have been used. In case of the inclusive reaction leading to $G_{M}^{n}$ we added $\pi$ - and $\rho$-like twobody exchange currents to the single nucleon current. Their effects were substantial. In the exclusive process only a single nucleon current operator has been used, which leaves room for improvement. In both cases a strictly nonrelativistic formulation has been used, which also should be improved. Our values for $G_{M}^{n}$ and $G_{E}^{n}$ agree well with the values extracted from processes on the deuteron.

From $3 \mathrm{~N}$ scattering it is known that the most modern data-equivalent $\mathrm{NN}$ forces lead in nearly all cases to results, which are very close together. We consider this robustness to be an important insight which gives confidence to those choices of the $3 \mathrm{~N}$ Hamiltonian. In the case of photon-induced processes a corresponding verification of robustness against interchanges of NN forces and consistent MEC's is still missing. This refers not only to the $3 \mathrm{~N}$ system but to the $2 \mathrm{~N}$ system as well. Also generally accepted and feasible relativistic formalisms have still to be worked out.

\section{ACKNOWLEDGMENTS}

This work was supported by the Deutsche Forschungsgemeinschaft (J.G. and H.K.), the Polish Committee for Scientific Research under Grant No. 2P03B03914 and the Science and Technology Cooperation Germany-Poland. One of us (W.G.) would like to thank the Foundation for Polish Science for the financial support during his stay in Cracow. We would like to thank Dr. H. Gao and Dr. D. Dutta for providing us with detailed information about their experiment and data analysis. The numerical calculations have been performed on the PVP machines of the NERSC, USA, on the Cray T90 of the NIC in Jülich, Germany, and on the VPP300 machine of the RWTH in Aachen, Germany. 


\section{REFERENCES}

[1] B. Blankleider, R. M. Woloshyn, Phys. Rev. C29, 538 (1984).

[2] J. L. Friar, B. F. Gibson, G. L. Payne, A. M. Bernstein, T. E. Chupp, Phys. Rev. C42, 2310 (1990).

[3] C.W. de Jager, Bates 25: Celebrating 25 years of beam to experiment, eds. T.W. Donnelly and W. Turchinetz, AIP conf. proc. 520, p. 225.

[4] D. Rohe et al., Phys. Rev. Lett. 83, 4257 (1999).

[5] I. Passchier et al., Phys. Rev. Lett. 82, 4988 (1999).

[6] C. Herberg et al., Eur. Phys. J. A 5, 131 (1999).

[7] H. Anklin et al., Phys. Lett. B336, 313 (1994).

[8] H. Anklin et al., Phys. Lett. B428, 248 (1998).

[9] A. Kievsky, E. Pace, G. Salme, M. Viviani, Phys. Rev. C56, 64 (1997);

C. Ciofi degli Atti, E. Pace, G. Salme, Phys. Rev. C46, R1591 (1992);

C. Ciofi degli Atti, E. Pace, G. Salme, Phys. Rev. C51, 1108 (1995);

R. W. Schultze, P. U. Sauer, Phys. Rev. C48, 38 (1993).

[10] W. Xu et al., accepted for publication in Phys. Rev. Lett.

[11] J. Becker and P. Grabmayr, private communication.

[12] J. Becker, PhD thesis, Johannes Gutenberg University, Mainz, 1997.

[13] J. Becker et al., Eur. Phys. J. A 6, 329 (1999).

[14] S. Ishikawa, J. Golak, H. Witała, H. Kamada, W. Glöckle, D. Hüber, Phys. Rev. C57, 39 (1998).

[15] G. Ziemer, PhD thesis, Ruhr-Universität Bochum, in preparation.

[16] V. V. Kotlyar, H. Kamada, J. Golak, W. Glöckle, Few Body Syst. 28, 35 (2000).

[17] D. O. Riska, Phys. Scr. 31, 107 (1985); 31, 471 (1985).

[18] R. B. Wiringa, V. G. J. Stoks, R. Schiavilla, Phys. Rev. C51, 38 (1995).

[19] J. Golak, H. Kamada, H. Witała, W. Glöckle, J. Kuroś, R. Skibiński, V. V. Kotlyar, K. Sagara, H. Akiyoshi, submitted to Phys. Rev. C.

[20] R. Machleidt, Adv. Nucl. Phys. 19 (1989) 189.

[21] J. Golak, H. Kamada, H. Witała, W. Glöckle, and S. Ishikawa, Phys. Rev. C51, 1638 (1995).

[22] W. Glöckle, H. Witała, D. Hüber, H. Kamada and J. Golak, Phys. Rep. 274, 107 (1996).

[23] T. W. Donnelly, A. S. Raskin, Ann. Phys. 169, 247 (1986).

[24] M. Gari and W. Krümpelmann, Phys. Lett. B173, 10 (1989); Phys. Lett. B274, 159 (1992); Erratum: Phys. Lett. B282 (1992).

[25] G. Höhler, E. Pietarinen, I. Sabba-Stefanescu, F. Borkowski, G. G. Simon, V. H. Walther, and R. D. Wendling, Nucl. Phys. B114, 505 (1976).

[26] F. Xiong, in preparation.

[27] P. Markowitz et al., Phys. Rev. C48, R5 (1993).

[28] E. E. W. Bruins et al., Phys. Rev. Lett. 75, 21 (1995).

[29] H. Gao et al., Phys. Rev. C50, R546 (1994); H. Gao, Nucl. Phys. A631, 170c (1998). 


\section{TABLES}

\begin{tabular}{|c|c|c|}
\hline \hline \hline$Q^{2}(\mathrm{GeV} / \mathrm{c})^{2}$ & $G_{M}^{n} / G_{M}^{n}($ Dipole $)$ & Uncertainties \\
\hline 0.1 & 0.966 & $\pm 0.014 \pm 0.01$ \\
0.2 & 0.962 & $\pm 0.013 \pm 0.01$ \\
\hline \hline
\end{tabular}

TABLE I. $G_{M}^{n}$ as a function of $Q^{2}$, the uncertainties are statistical and systematic.

\begin{tabular}{|c|c|c|}
\hline \hline$\theta_{e}\left[^{\circ}\right]$ & $p_{n}^{\text {min }}[\mathrm{MeV} / \mathrm{c}]$ & $p_{n}^{\text {max }}[\mathrm{MeV} / \mathrm{c}]$ \\
\hline 39 & 500.31 & 598.68 \\
40 & 507.60 & 609.08 \\
41 & 515.14 & 619.88 \\
42 & 522.93 & 631.15 \\
43 & 530.99 & 642.90 \\
44 & 539.33 & 655.18 \\
45 & 547.98 & 668.00 \\
46 & 556.94 & 681.43 \\
47 & 566.24 & 695.52 \\
48 & 575.91 & 704.73 \\
49 & 585.95 & 714.23 \\
50 & 596.40 & 724.04 \\
51 & 607.28 & 734.18 \\
52 & 618.65 & 744.66 \\
53 & 630.51 & 755.50 \\
54 & 642.90 & 766.74 \\
55 & 651.02 & 772.61 \\
56 & 659.38 & 778.61 \\
57 & 668.00 & 784.69 \\
58 & 676.90 & 790.91 \\
59 & 686.06 & 797.24 \\
\hline \hline
\end{tabular}

TABLE II. Intervals for the neutron momenta. 


\begin{tabular}{|l|ccc|}
\hline \hline & & $Q^{2}\left[(\mathrm{GeV} / \mathrm{c})^{2}\right]$ & \\
& 0.30 & 0.35 & 0.40 \\
\hline PWIA & $0.0441 \pm 0.0035$ & $0.0465 \pm 0.0038$ & $0.0484 \pm 0.0038$ \\
PWIAS & $0.0455 \pm 0.0035$ & $0.0480 \pm 0.0038$ & $0.0499 \pm 0.0038$ \\
FSI23 & $0.0406 \pm 0.0035$ & $0.0428 \pm 0.0037$ & $0.0446 \pm 0.0038$ \\
FSI & $0.0474 \pm 0.0036$ & $0.0499 \pm 0.0038$ & $0.0520 \pm 0.0038$ \\
\hline \hline & 0.45 & $Q^{2}\left[(G e V / c)^{2}\right]$ & 0.55 \\
& $0.0500 \pm 0.0039$ & 0.50 & $0.0523 \pm 0.0049$ \\
& $0.0515 \pm 0.0038$ & $0.0512 \pm 0.0043$ & $0.0539 \pm 0.0049$ \\
PWIA & $0.0460 \pm 0.0038$ & $0.0529 \pm 0.0043$ & $0.0482 \pm 0.0048$ \\
PWIAS & $0.0536 \pm 0.0039$ & $0.0472 \pm 0.0043$ & $0.0561 \pm 0.0050$ \\
FSI23 & & $0.0550 \pm 0.0044$ & \\
FSI & &
\end{tabular}

TABLE III. Extracted averaged $G_{E}^{n}$ values (see text) obtained for different assumptions about the final state. The uncertainties arise from the spread in the form factor parametrization and the experimental error. 


\section{FIGURES}

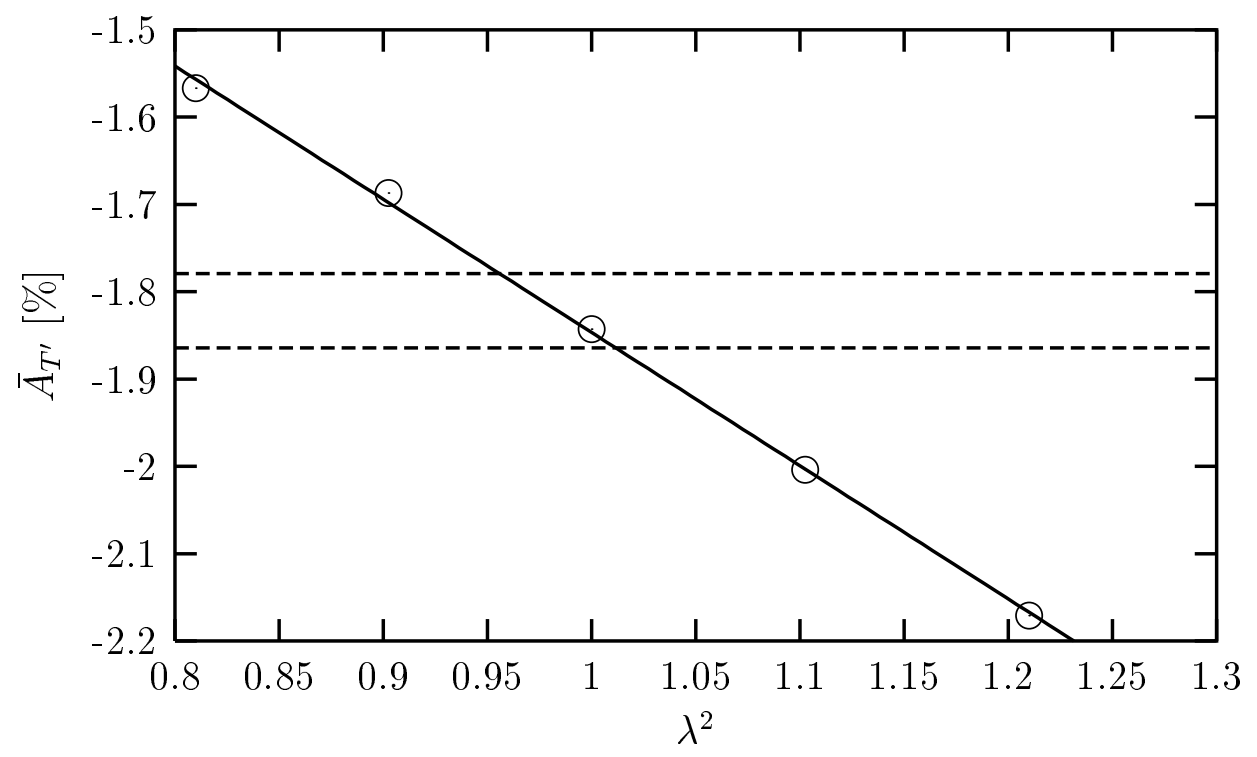

FIG. 1. The averaged asymmetry $\bar{A}_{T^{\prime}}$ of Eq. (6) around the quasielastic peak against the $\lambda^{2}$-factor for $Q^{2}=0.1 \mathrm{GeV}^{2} / c^{2}$. The solid curve is a result of a fit. Dashed curves show the experimental bounds for $A_{T^{\prime}}$.

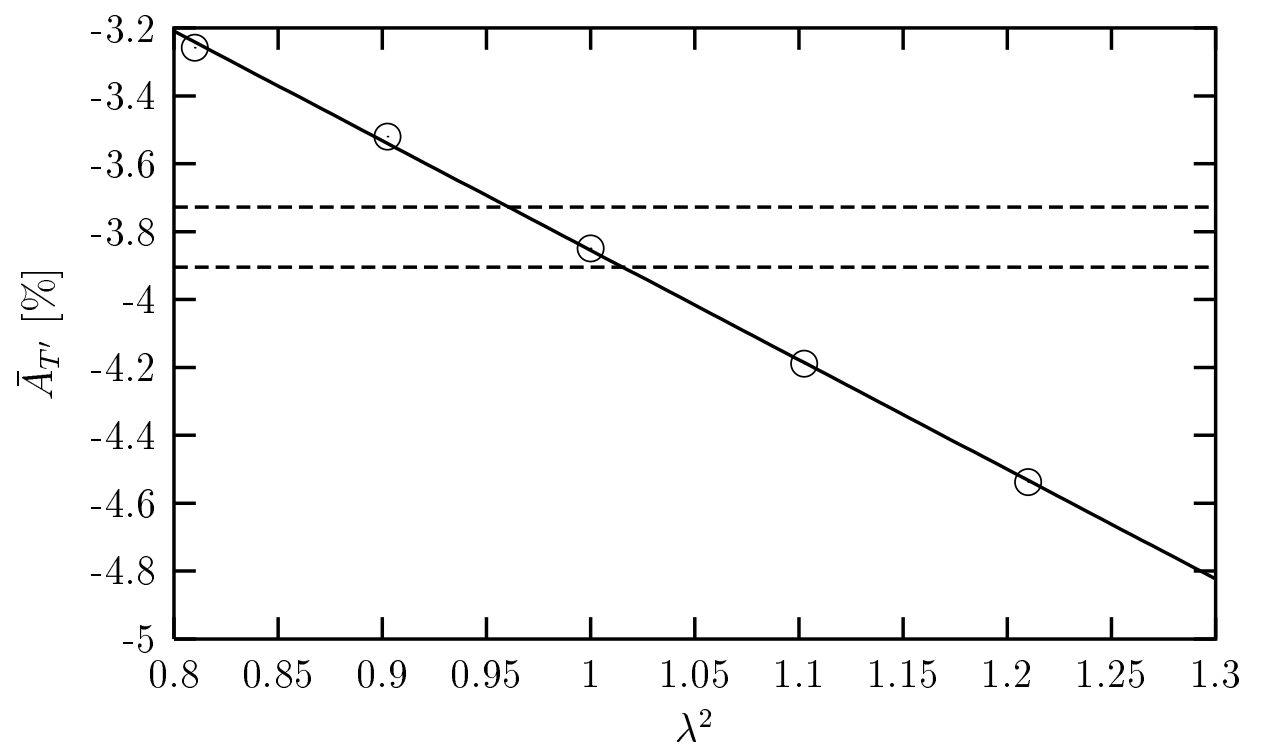

FIG. 2. The same as in Fig. 1 for $Q^{2}=0.2 \mathrm{GeV}^{2} / c^{2}$. 


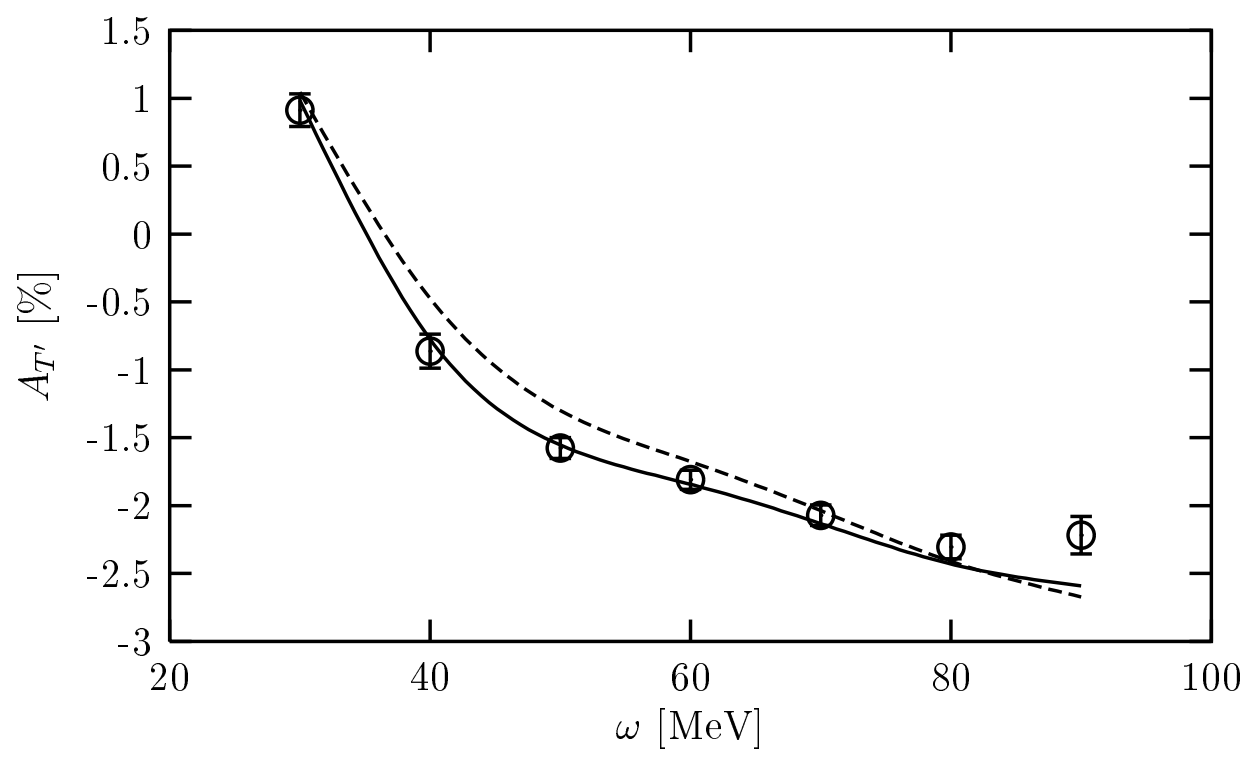

FIG. 3. The asymmetry $A_{T^{\prime}}$ against the energy transfer $\omega$ for $Q^{2}=0.1 \mathrm{GeV}^{2} / c^{2}$. The curves describe full (averaged) Bonn B predictions with the single nucleon current (dashed) and with the single nucleon current plus the $\pi$ - and $\rho$-MEC (solid). Data are from [10].

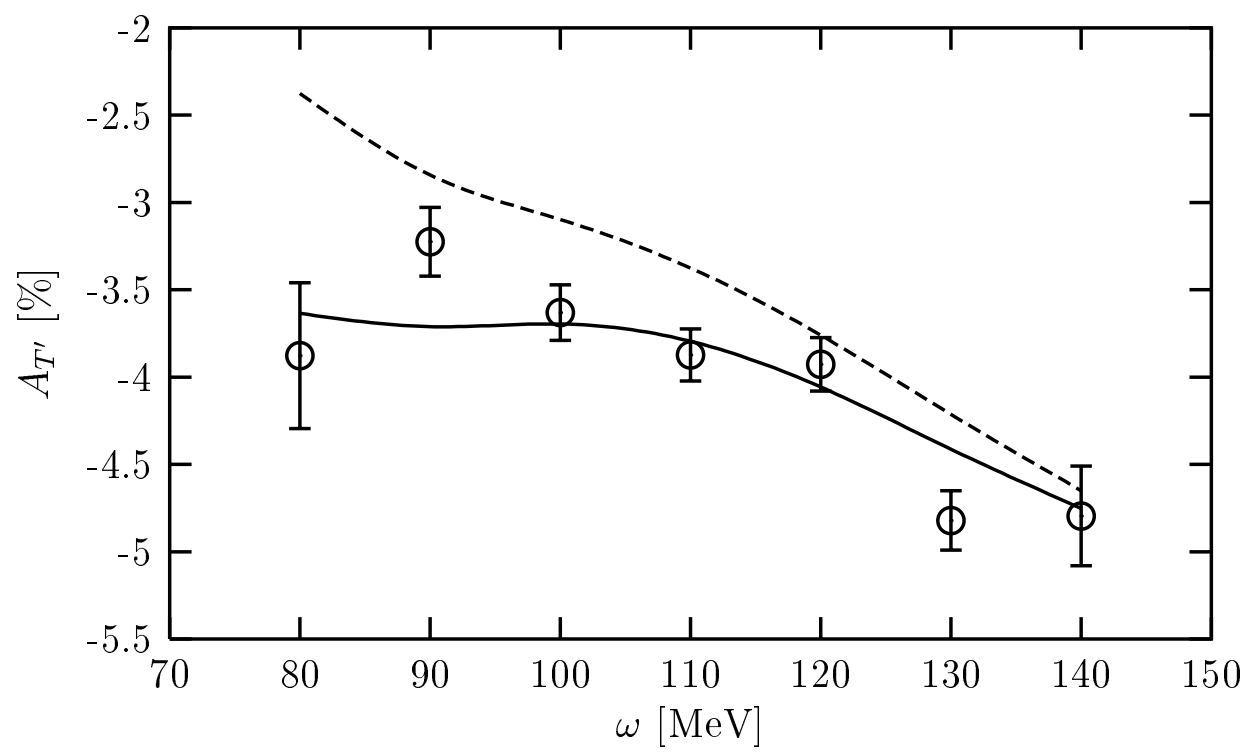

FIG. 4. The same as in Fig. 3 for $Q^{2}=0.2 \mathrm{GeV}^{2} / c^{2}$. 


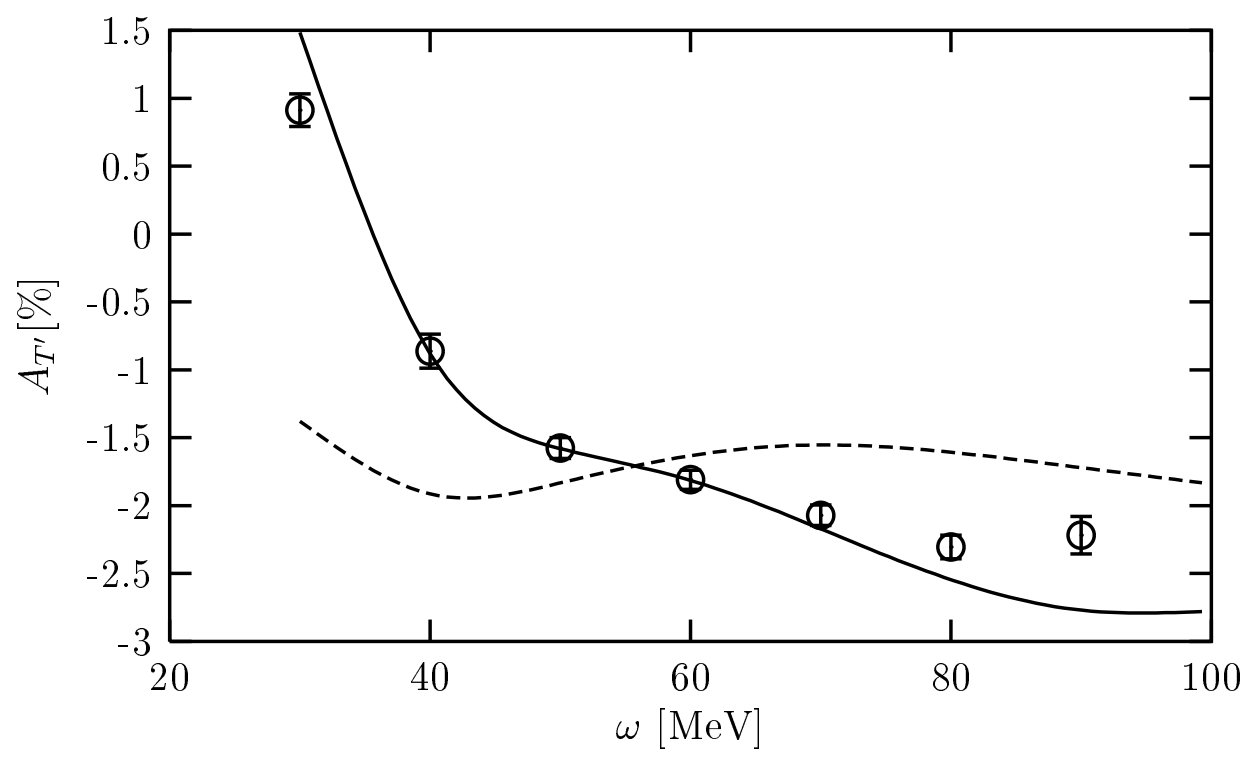

FIG. 5. The asymmetry $A_{T^{\prime}}$ against the energy transfer $\omega$ for $Q^{2}=0.1 \mathrm{GeV}^{2} / c^{2}$. The curves describe point geometry results obtained with the AV18 potential: PWIA (dashed) and the full prediction (solid). In both cases the single nucleon current plus the $\pi$ - and $\rho$-MEC is used. Data are from [10].

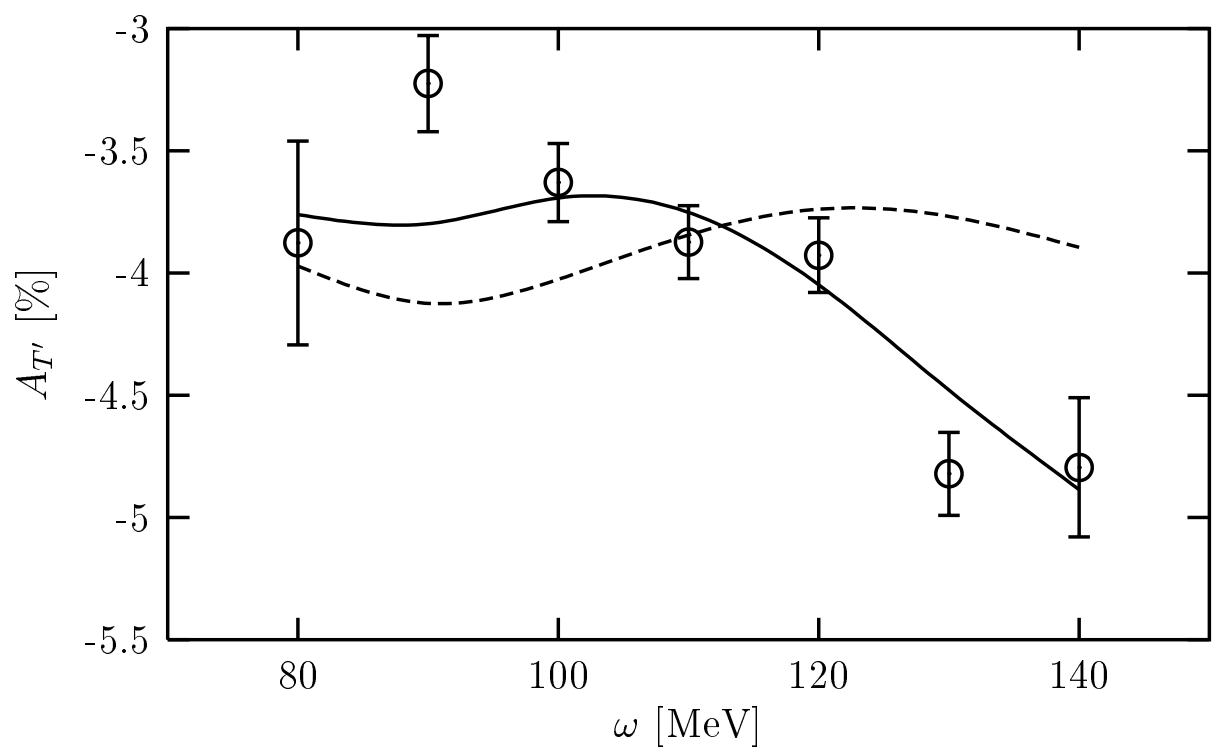

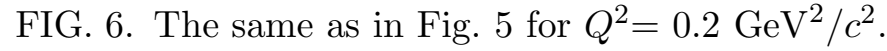




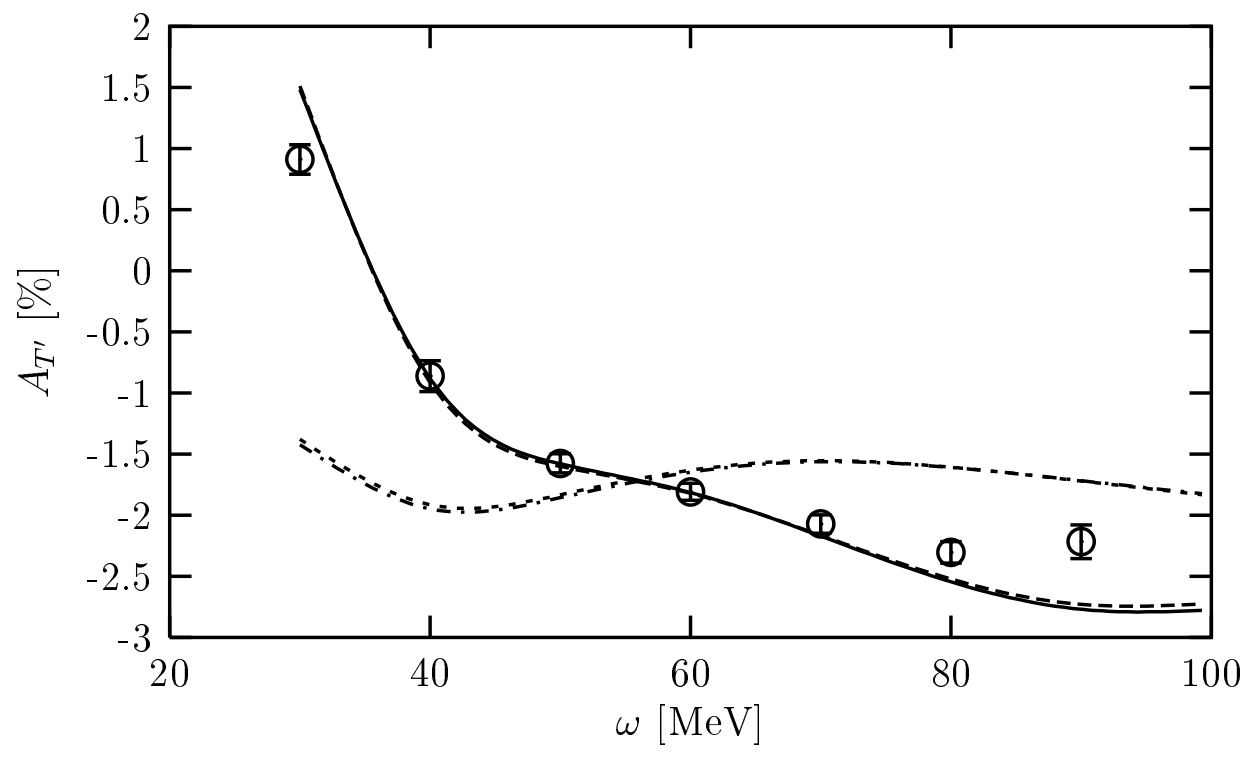

FIG. 7. The asymmetry $A_{T^{\prime}}$ against the energy transfer $\omega$ for $Q^{2}=0.1 \mathrm{GeV}^{2} / c^{2}$. The curves describe PWIA point geometry results with $G_{E}^{n}=0$ (dashed-dotted), and $G_{E}^{n} \neq 0$ (dotted), full point geometry results with $G_{E}^{n}=0$ (dashed) and $G_{E}^{n} \neq 0$ (solid). All results are obtained with the AV18 potential. The single nucleon current plus the $\pi$ - and $\rho$-MEC is used. Data are from [10].

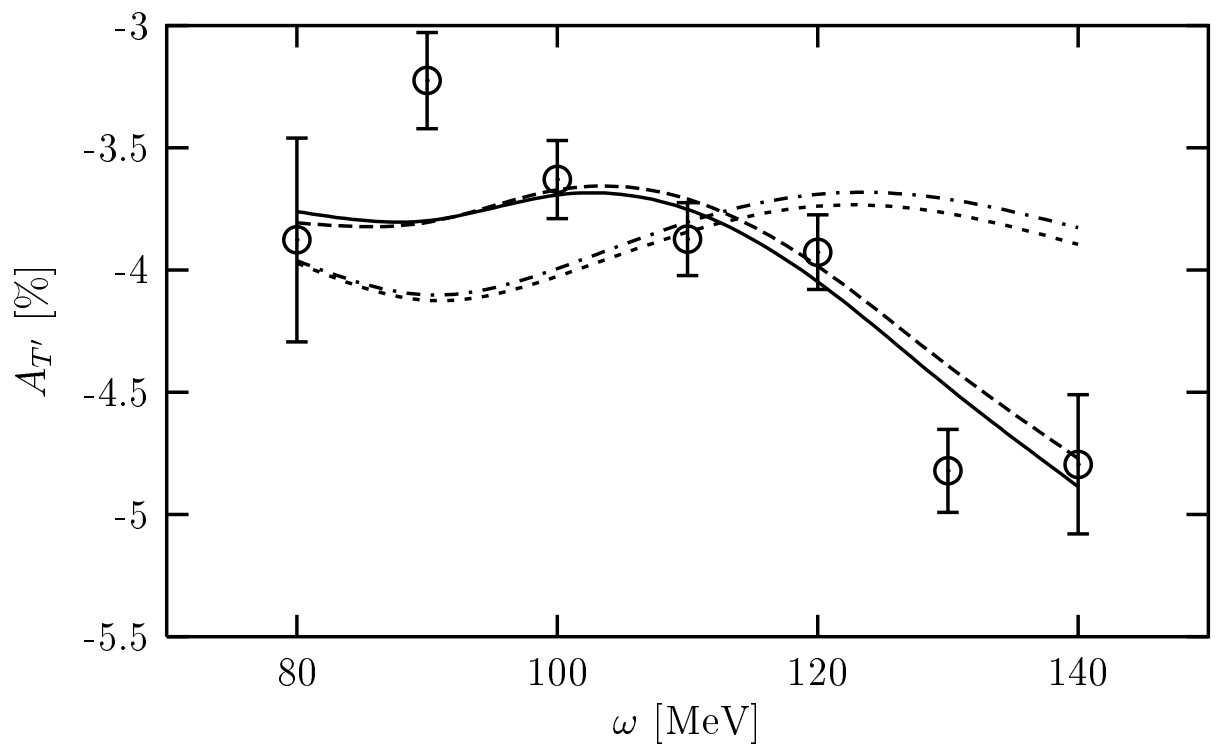

FIG. 8. The same as in Fig. 7 for $Q^{2}=0.2 \mathrm{GeV}^{2} / c^{2}$. 


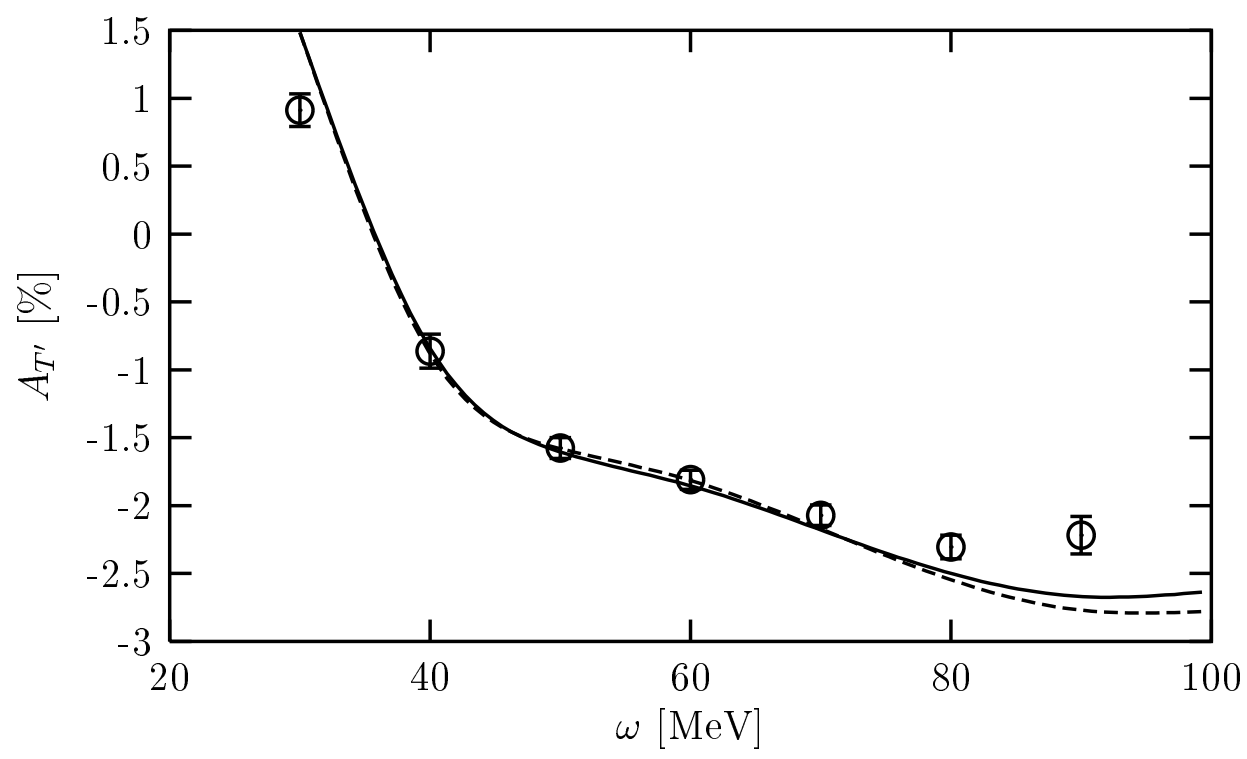

FIG. 9. The asymmetry $A_{T^{\prime}}$ against the energy transfer $\omega$ for $Q^{2}=0.1 \mathrm{GeV}^{2} / c^{2}$. The curves describe full point geometry results obtained with the AV18 potential (dashed) and with the Bonn B potential (solid). In both cases the single nucleon current plus the $\pi$ - and $\rho$-MEC are used. Data are from [10].

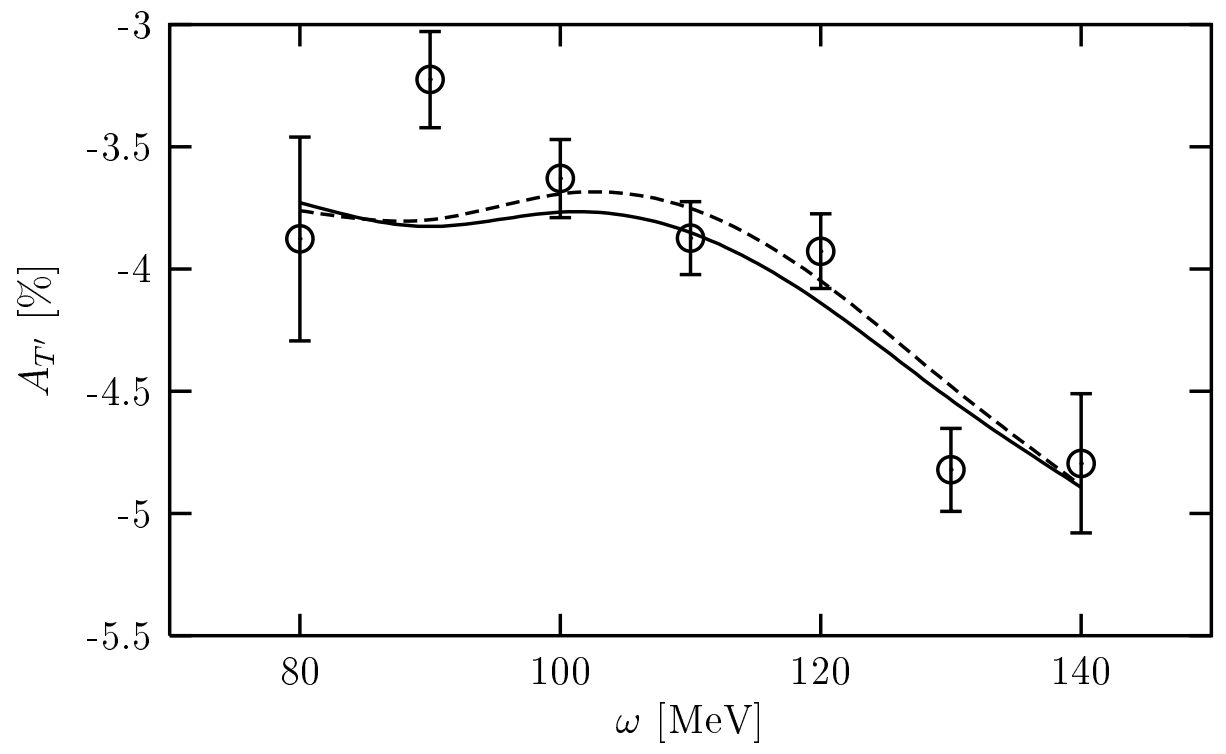

FIG. 10. The same as in Fig. 9 for $Q^{2}=0.2 \mathrm{GeV}^{2} / c^{2}$. 


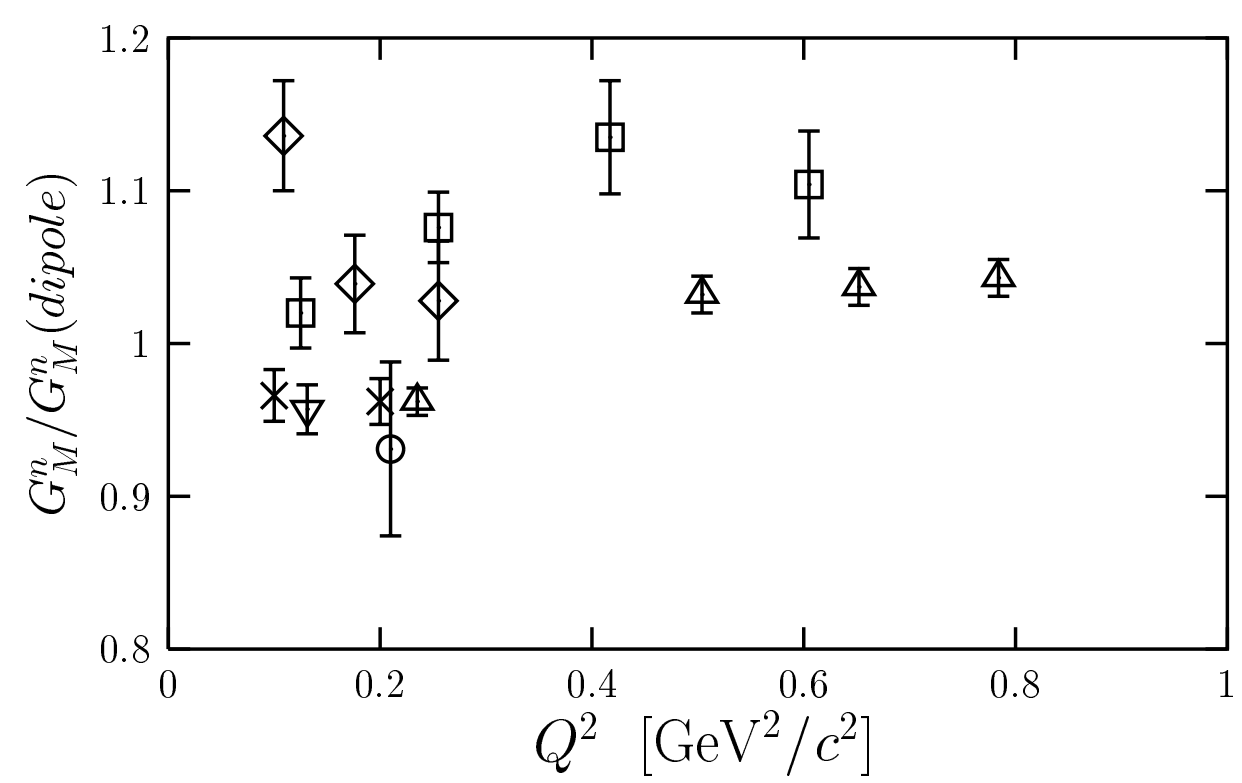

FIG. 11. $G_{M}^{n}$-values extracted from different measurements on the deuteron ( [27] $(\diamond),[7](\nabla)$, [28] (口), [8] $(\triangle))$ and on ${ }^{3} \mathrm{He}([29](\bigcirc)$, 10] $(\times))$.

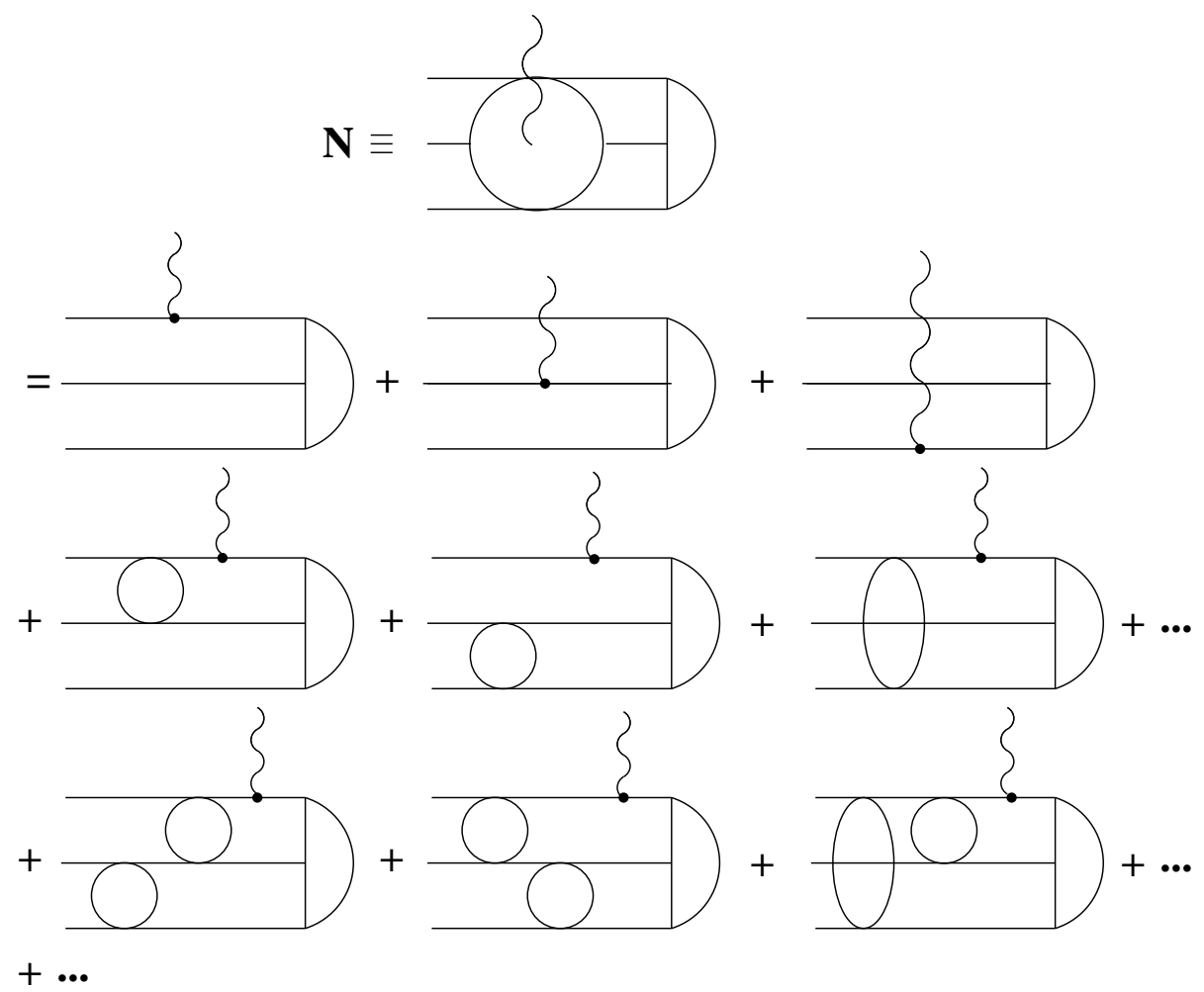

FIG. 12. The multiple rescattering series for the process ${ }^{3} \mathrm{He}(\mathrm{e}, \mathrm{e}$ 'n). The half moon stands for the ${ }^{3} \mathrm{He}$ state, the wavy line for the photon, horizontal lines for freely propagating nucleons and the ovals for NN t-matrices. The dots in the third line stand for processes, where the photon is absorbed on the other two nucleons. 


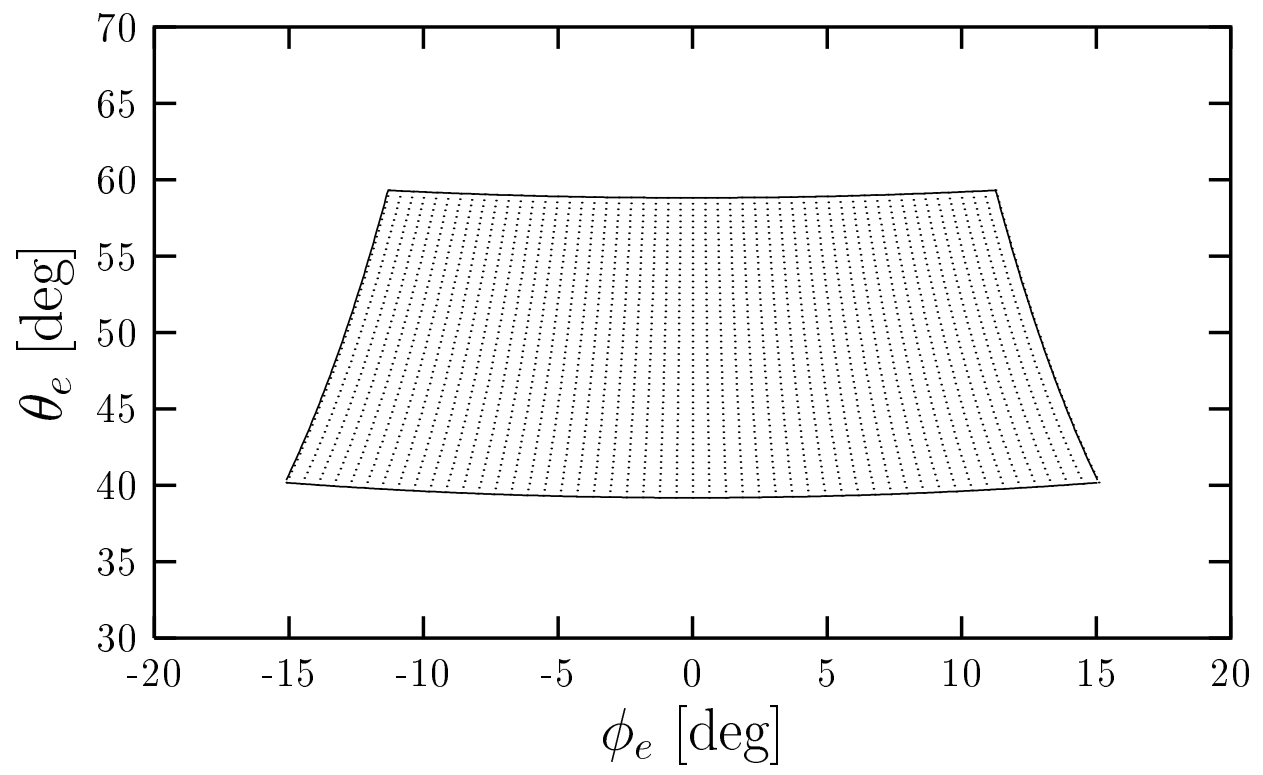

FIG. 13. Angular acceptance of the electron detector.

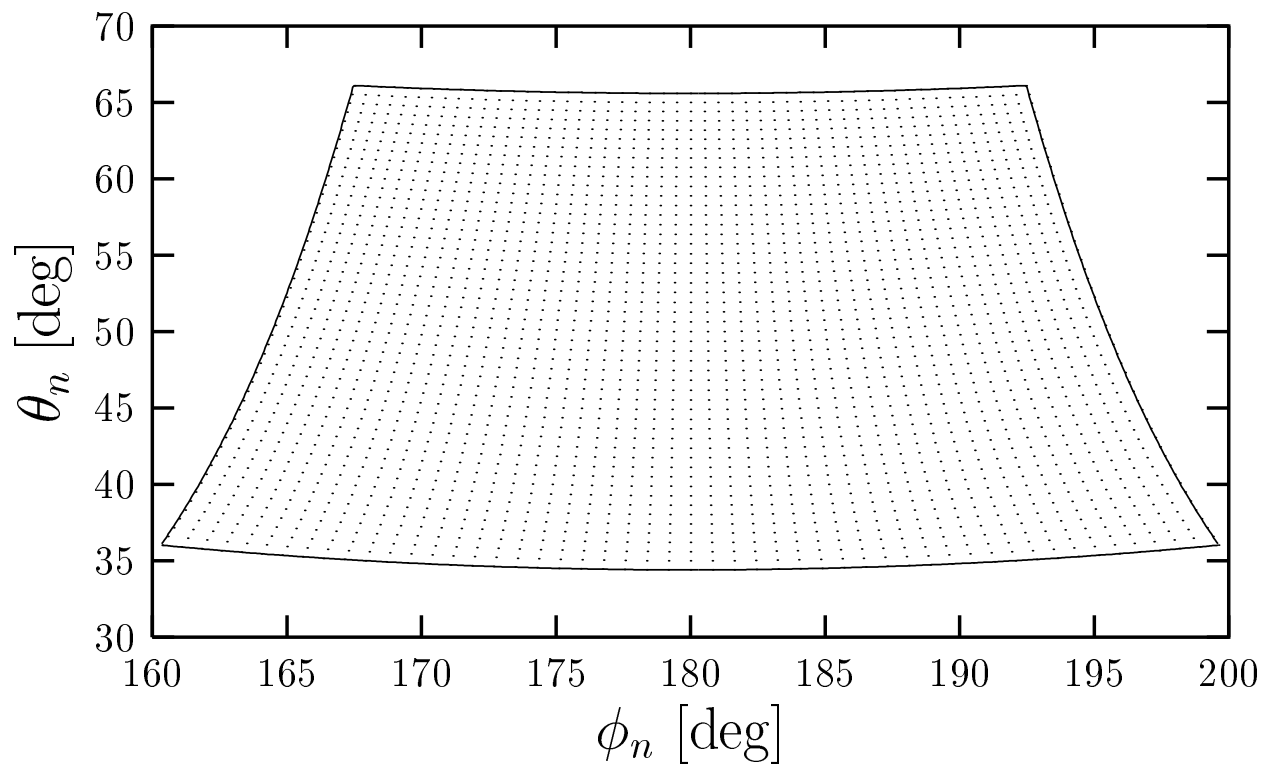

FIG. 14. Angular acceptance of the neutron detector. 


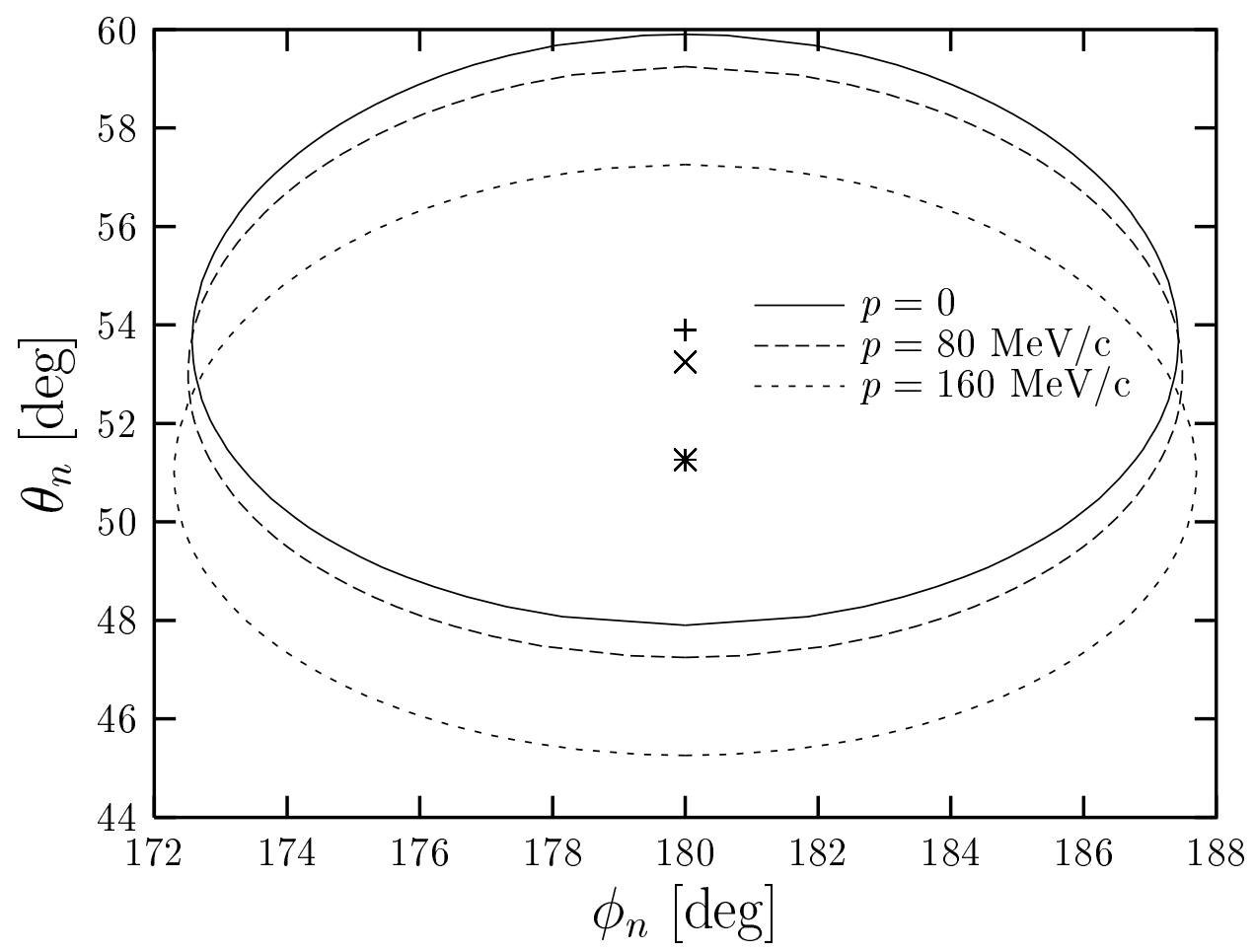

FIG. 15. Integration limits for $\hat{p}_{n}$ (solid curve) for the example $\theta_{e}=43^{\circ}, \phi_{e}=0^{\circ}$ and $p_{n}=$ $530 \mathrm{MeV} / \mathrm{c}$ together with the direction of the photon + . The dashed and dotted curves are for $p$ $=80$ and $160 \mathrm{MeV} / \mathrm{c}$ and the corresponding directions of the photon are given by $\times$ and $*$.

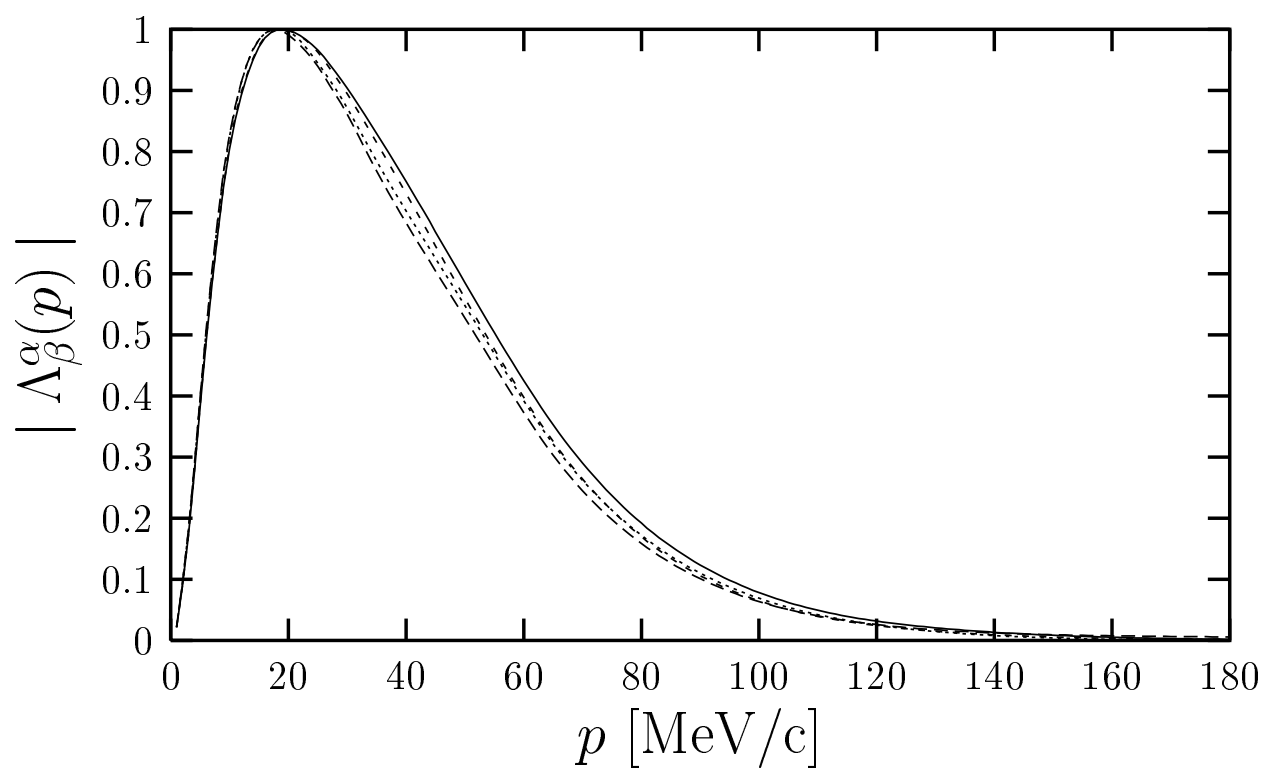

FIG. 16. The magnitudes of the $\Lambda_{\|}^{T^{\prime}}(p)$ (solid), $\Lambda_{\|}^{T L^{\prime}}(p)$ (long dashed), $\Lambda_{\perp}^{T^{\prime}}(p)$ (short dashed) and $\Lambda_{\perp}^{T L^{\prime}}(p)$ (dotted) amplitudes of Eq. (23) for full FSI as a function of $p$. They are all arbitrarily normalised to 1 at their maxima and correspond to the arbitrarily chosen values of $\theta_{e}=43^{\circ}, \phi_{e}$ $=0^{\circ}, \theta_{n}=53.9^{\circ}, \phi_{n}=180^{\circ}$ and $p_{n}=530 \mathrm{MeV} / \mathrm{c}$. 


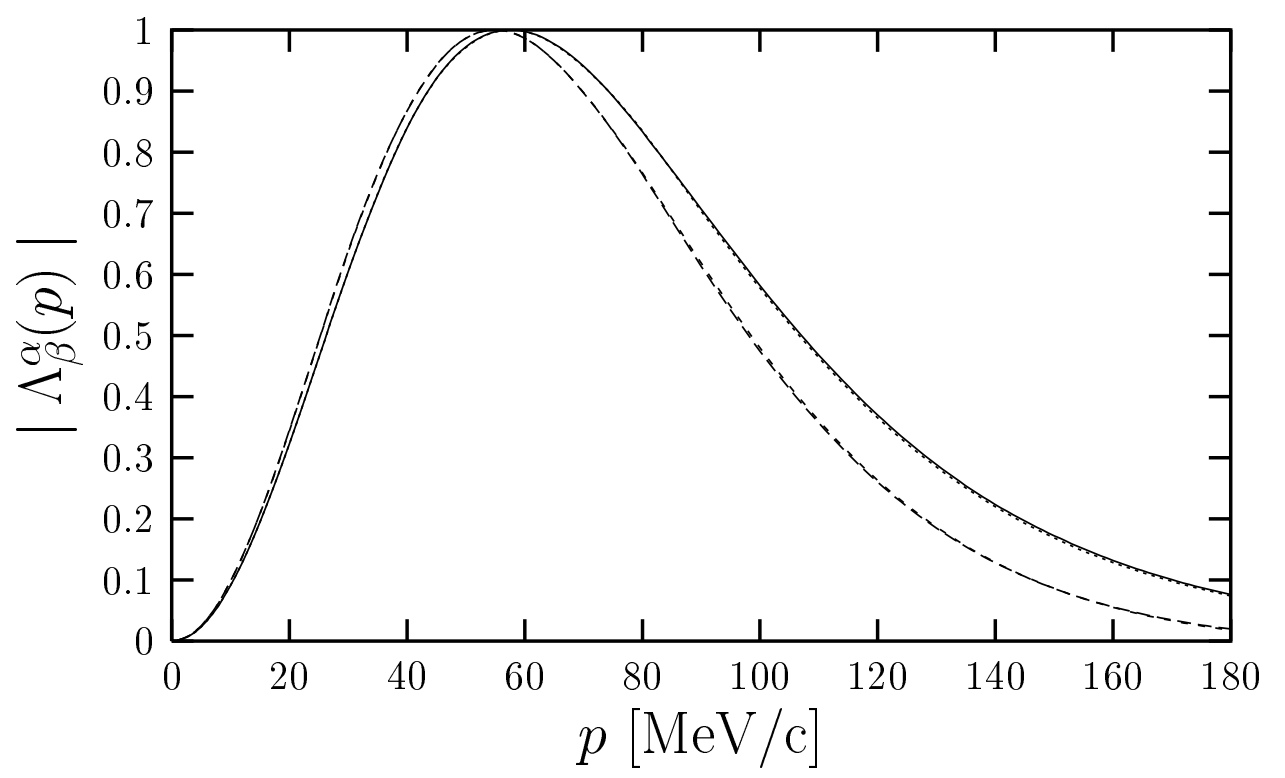

FIG. 17. The same as in Fig. 16 for PWIA.

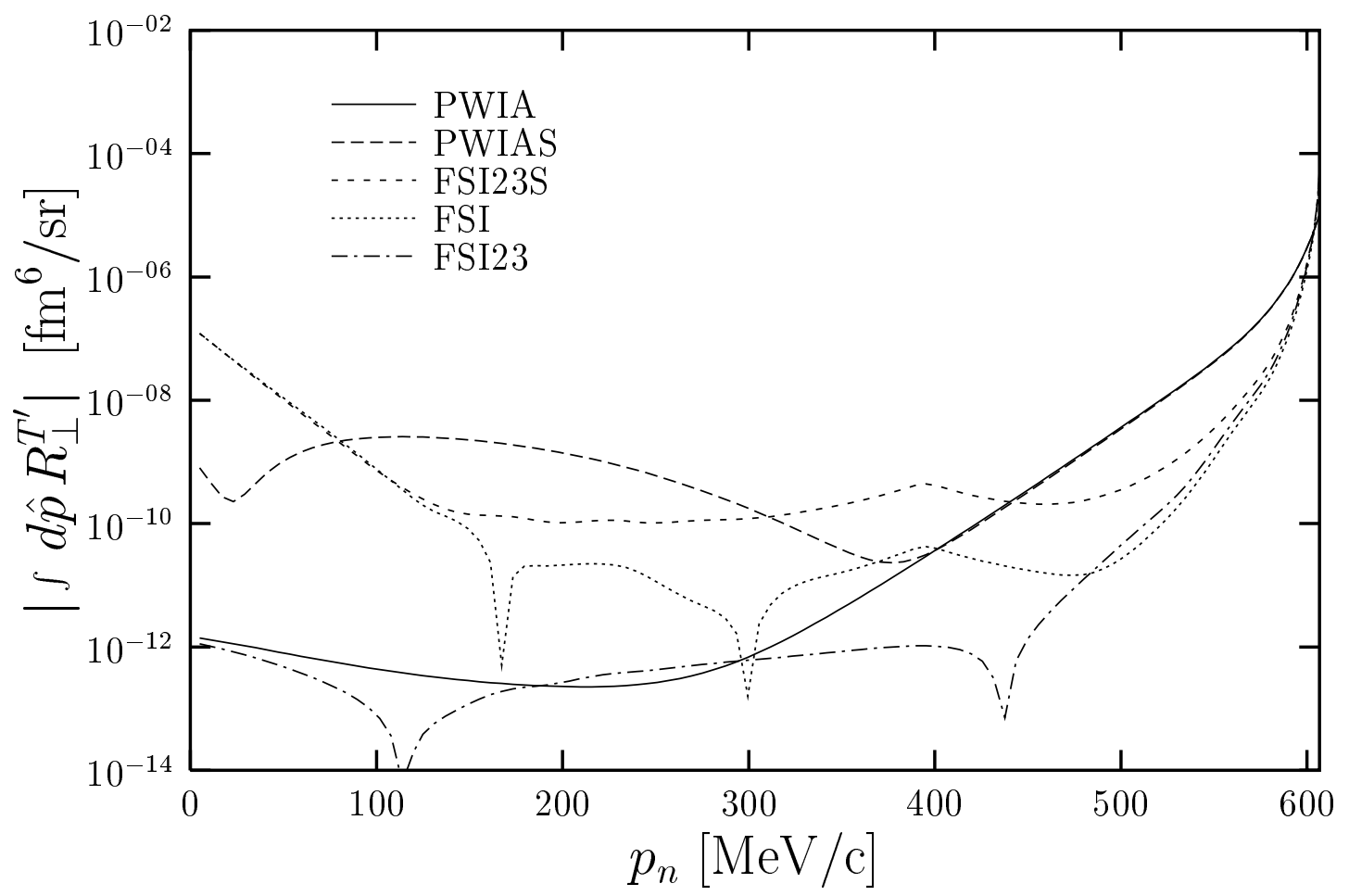

FIG. 18. FSI effects in the integrated response function $R^{T^{\prime}}$. 


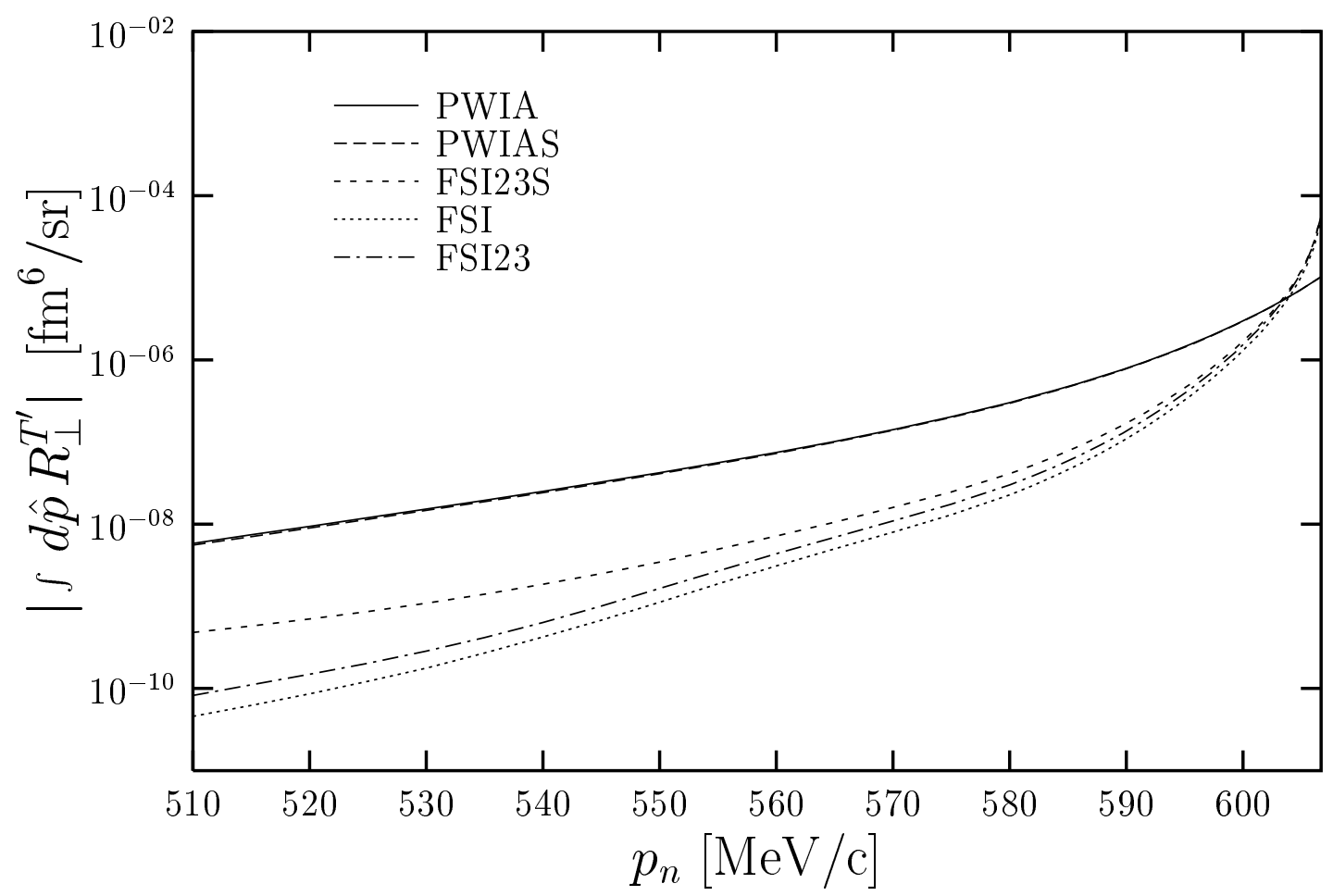

FIG. 19. The same as in Fig. 18 for the truncated $p_{n}$ region used in the analysis of the experiment [13].

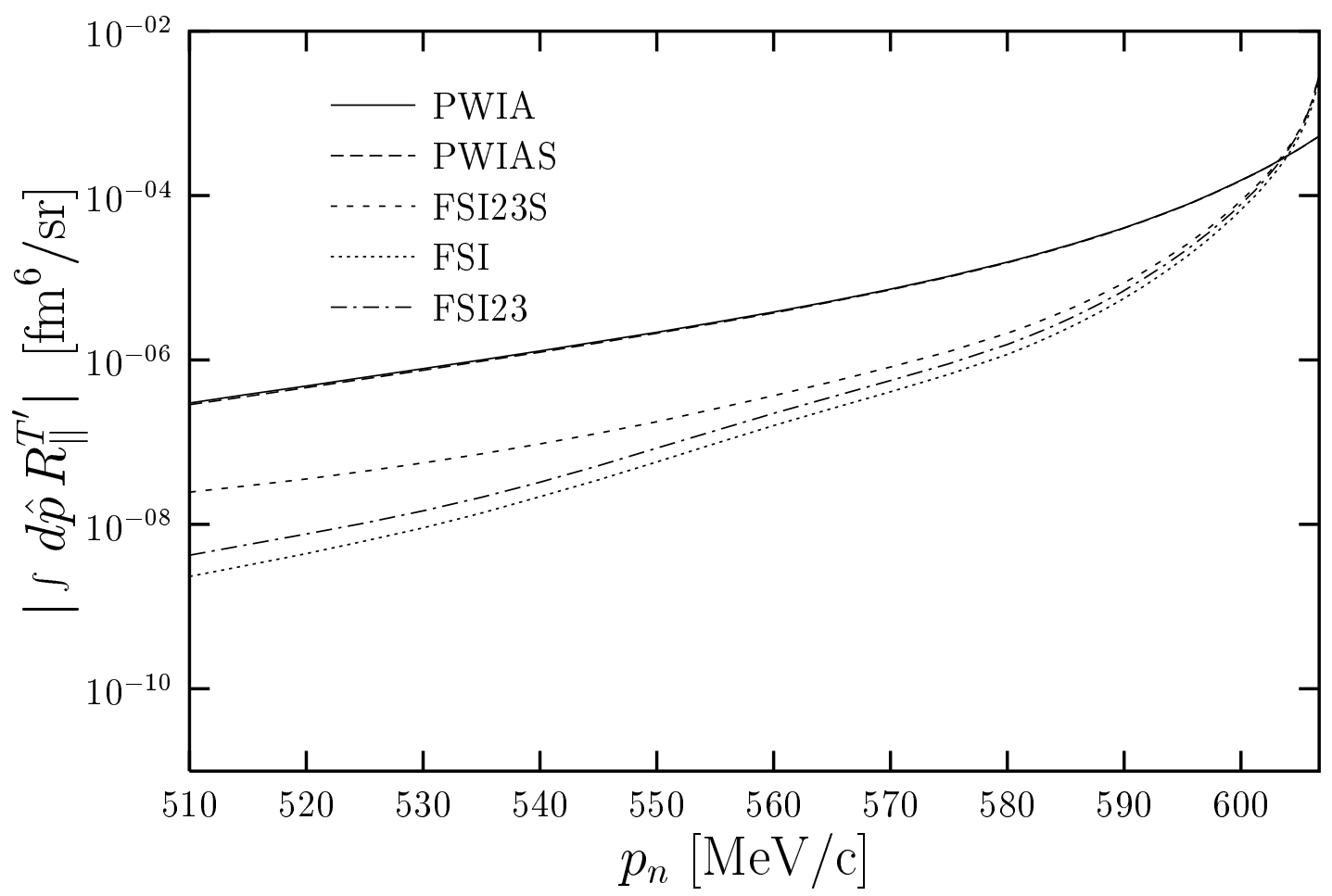

FIG. 20. The same as in Fig. 19 but for the parallel orientation of the target spin. 


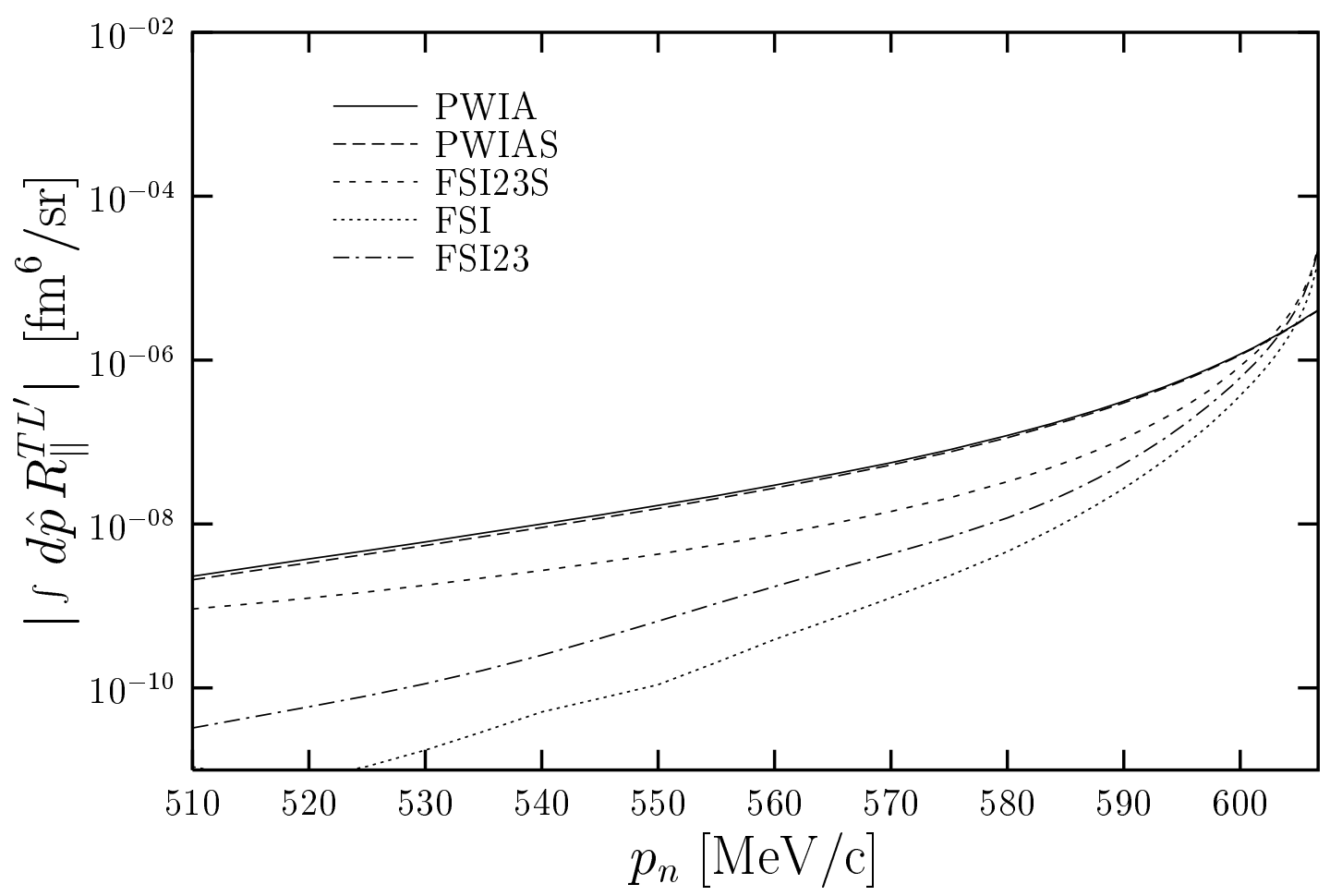

FIG. 21. The same as in Fig. 20 but for the response function $R^{T L^{\prime}}$.

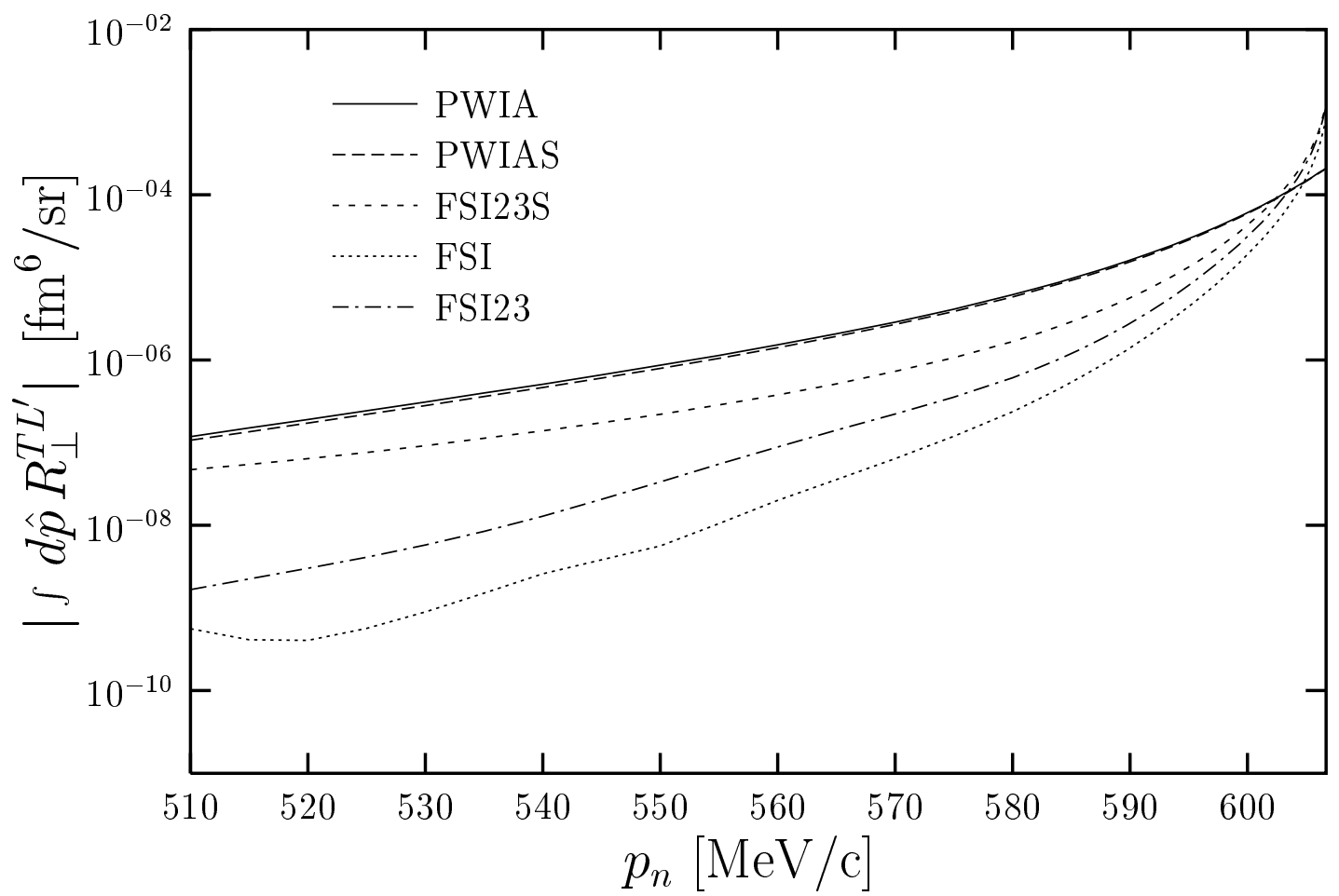

FIG. 22. The same as in Fig. 21 but for the parallel orientation of the target spin. 


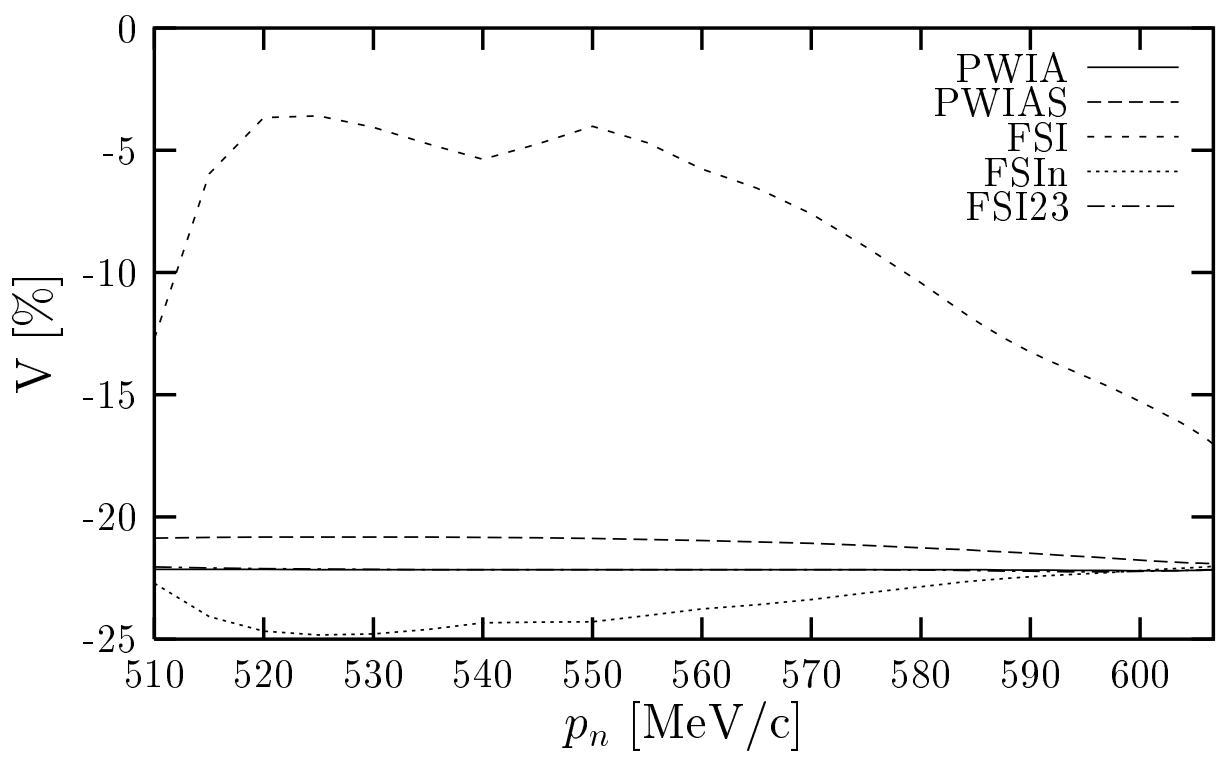

FIG. 23. Ratios $V$ for point geometry $\left(k_{0}^{\prime}=650 \mathrm{MeV} / \mathrm{c}, \theta_{e}=40^{\circ}, \theta_{n}=49.48^{\circ}, \theta_{\|}^{\star}=1.12^{\circ}, \theta_{\perp}^{\star}=\right.$ $88.88^{\circ},|\vec{Q}|=549.61 \mathrm{MeV} / \mathrm{c}$ ) for various treatments of the final state against $p_{n}$.

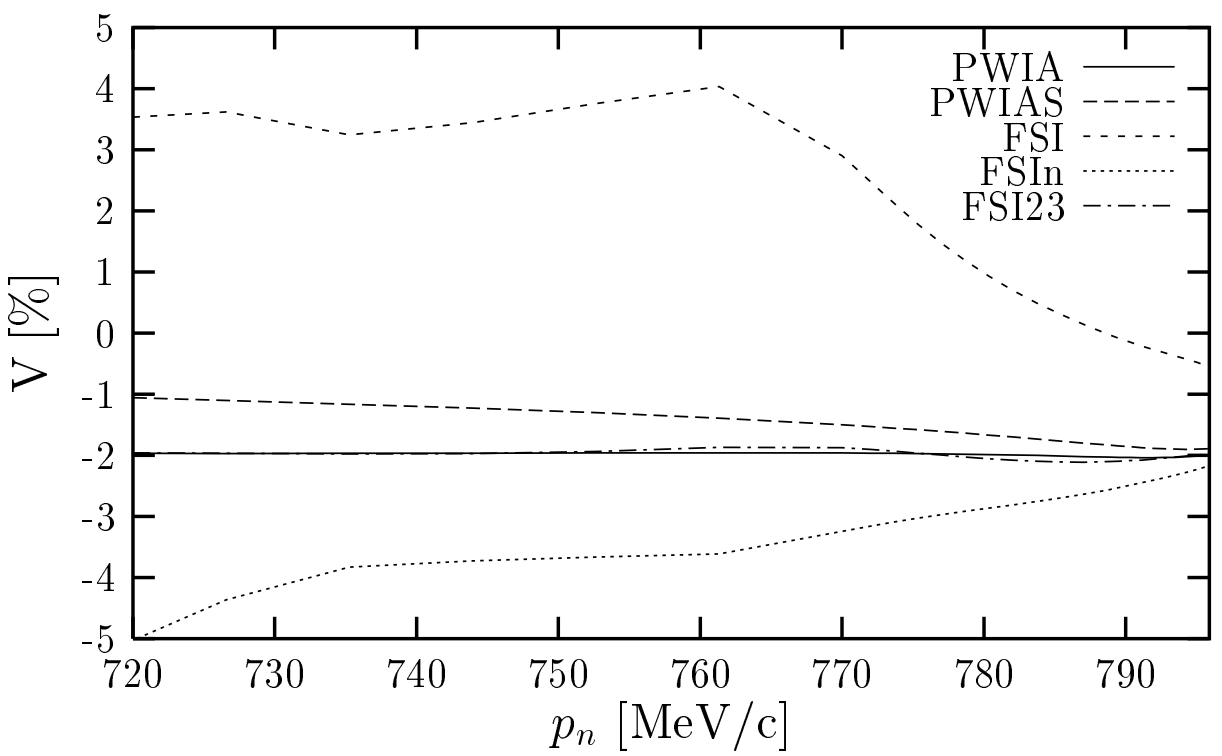

FIG. 24. The same as in Fig. 23 for $k_{0}^{\prime}=508 \mathrm{MeV} / \mathrm{c}, \theta_{e}=58^{\circ}, \theta_{n}=36.33^{\circ}, \theta_{\|}^{\star}=14.27^{\circ}, \theta_{\perp}^{\star}=$ $75.73^{\circ},|\vec{Q}|=727.16 \mathrm{MeV} / \mathrm{c}$. 


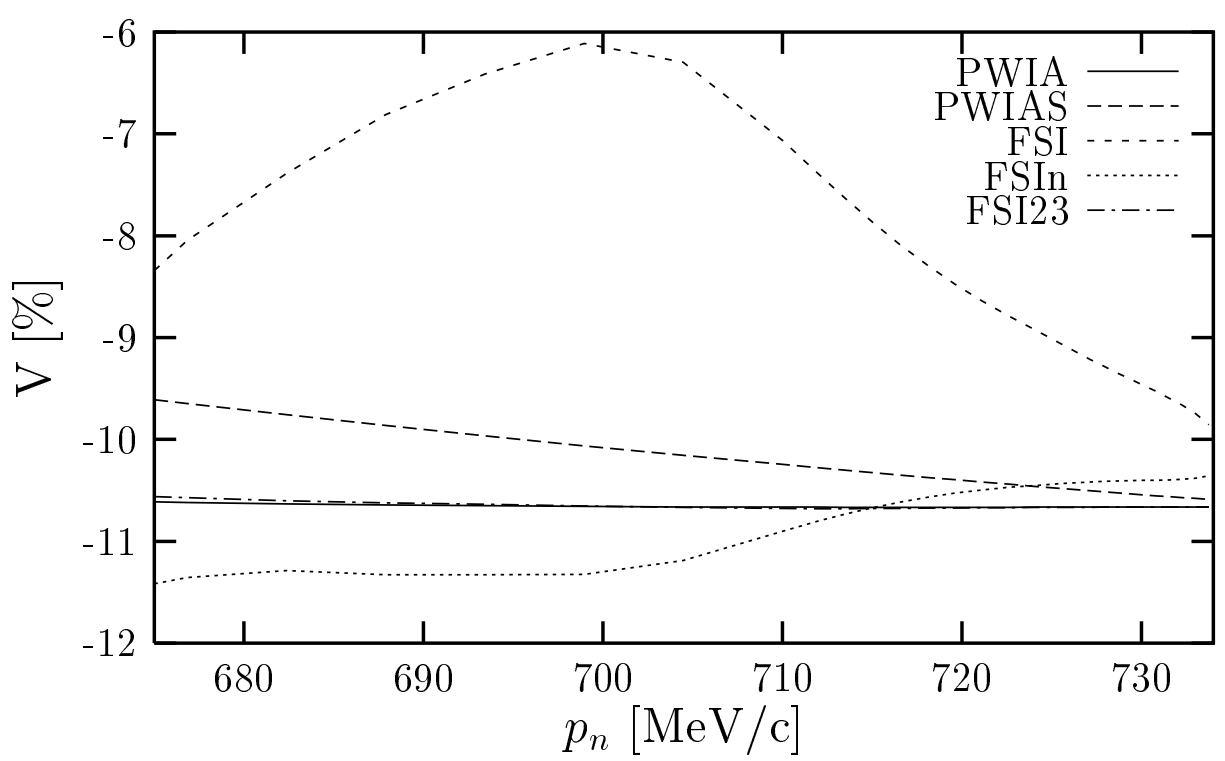

FIG. 25. The same as in Fig. 23 for $k_{0}^{\prime}=560 \mathrm{MeV} / \mathrm{c}, \theta_{e}=58^{\circ}, \theta_{n}=40.93^{\circ}, \theta_{\|}^{\star}=10.21^{\circ}, \theta_{\perp}^{\star}=$ $79.79^{\circ},|\vec{Q}|=732.92 \mathrm{MeV} / \mathrm{c}$.

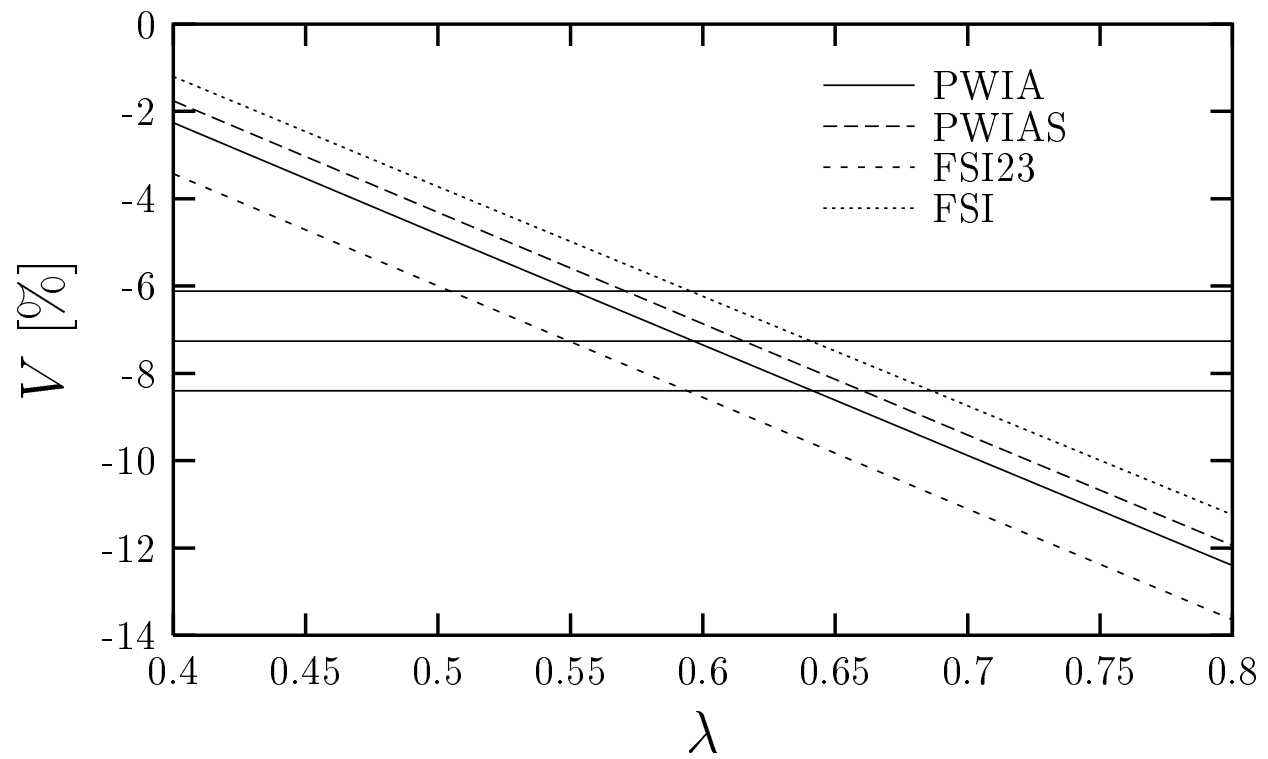

FIG. 26. Theoretical ratios $V$ against $\lambda$ in comparison to the experimental value including its error. 


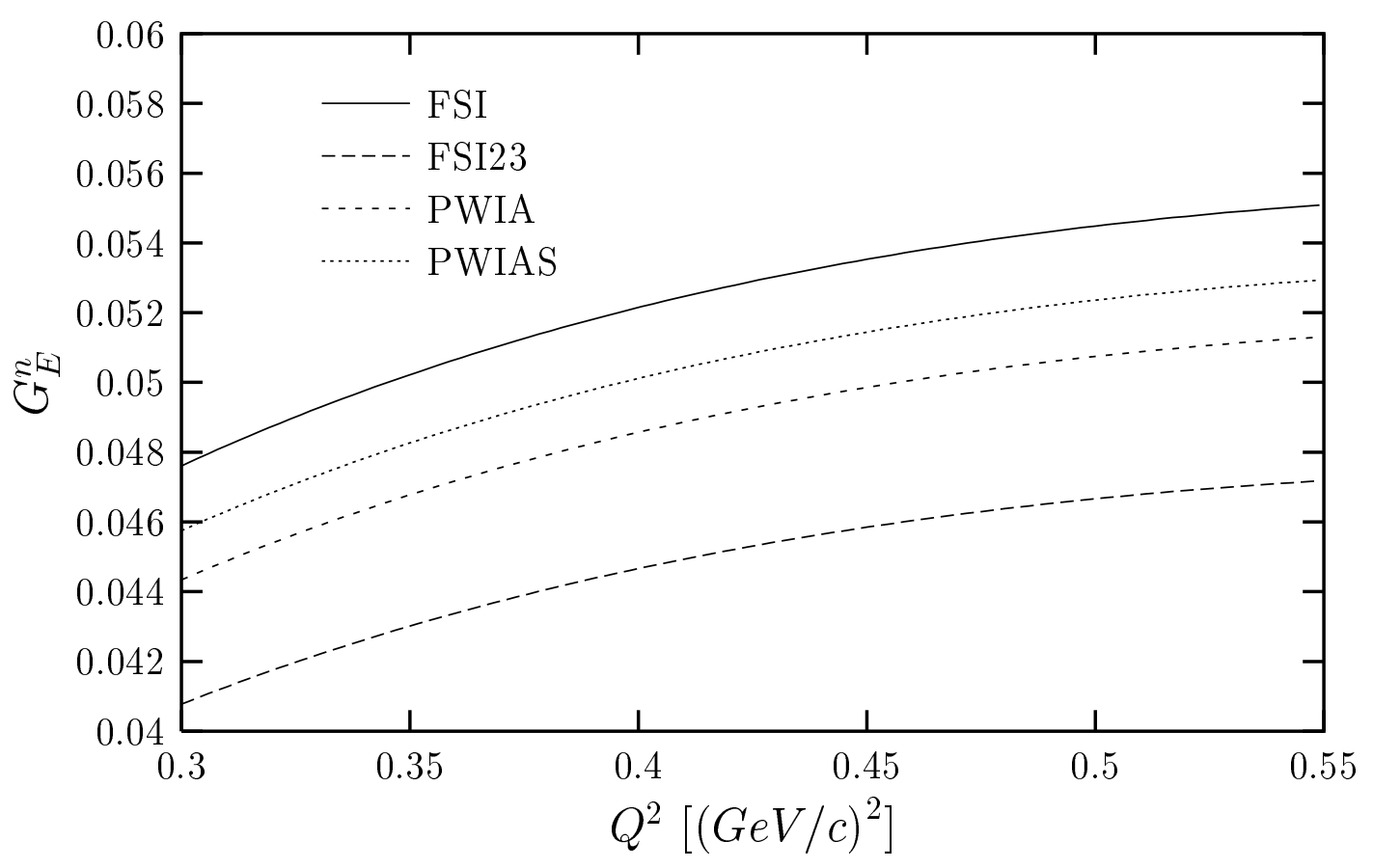

FIG. 27. Extracted $G_{E}^{n}$-values for different treatment of the final state.

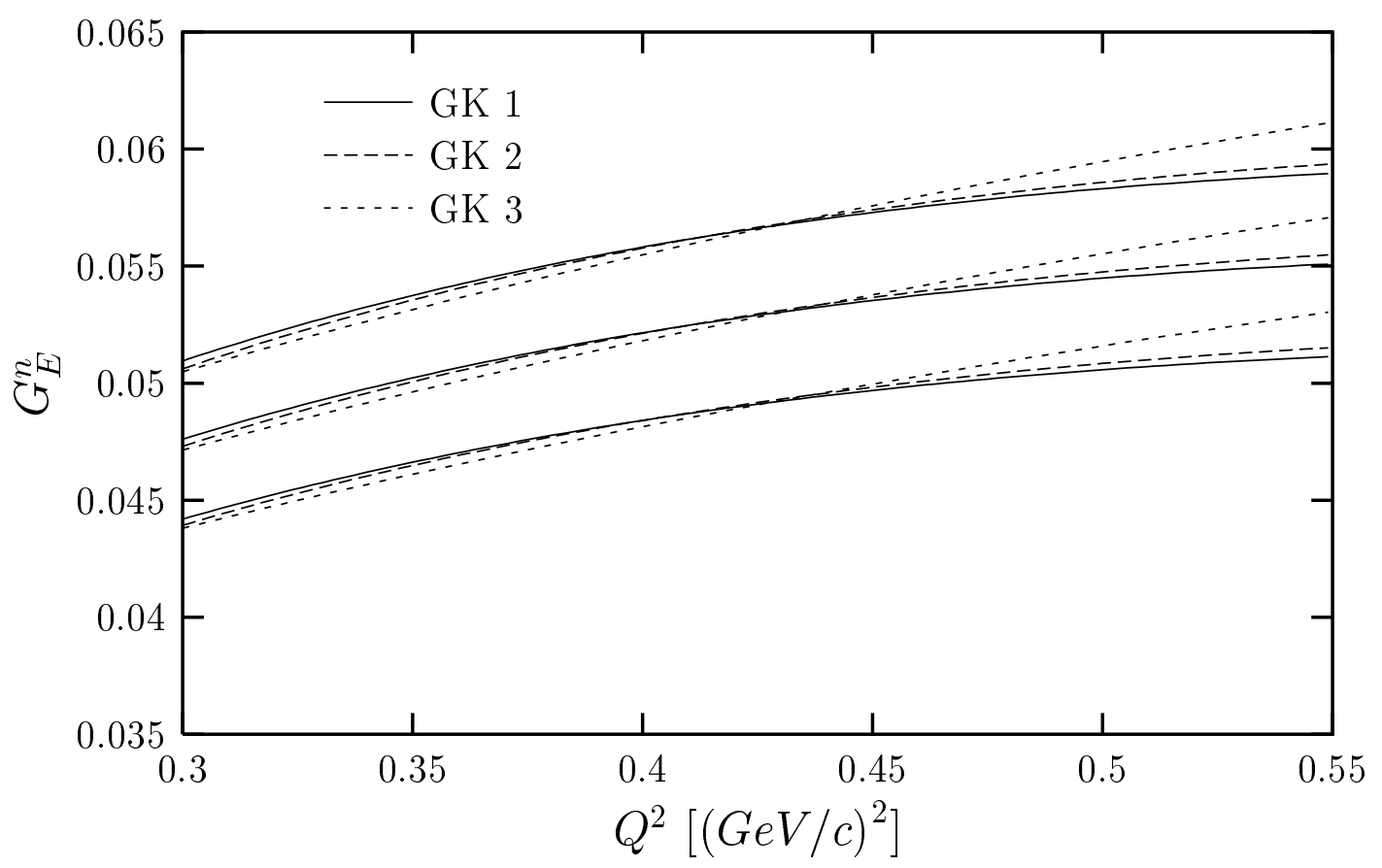

FIG. 28. The extracted $G_{E}^{n}$ for full FSI. The three separated curves correspond to the three $\lambda$-values from Fig. 26. The smaller spread of curves is due to the three different parametrisations of $\left.G_{E}^{n}\right|_{\text {model }}$. 


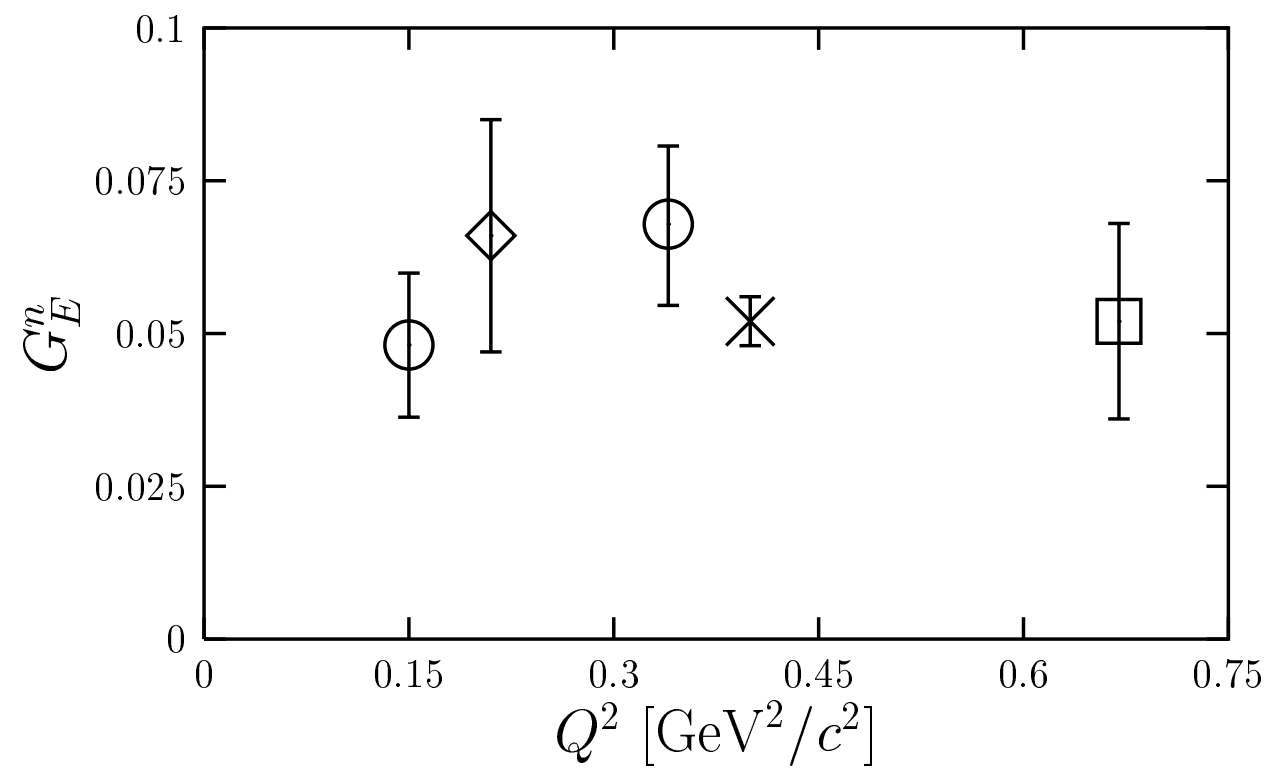

FIG. 29. $G_{E}^{n}$-values extracted from ${ }^{3} \mathrm{He}$ (this work $(\times)$, 邨 $(\square)$ ) and from processes on the deuteron $($ 5 $(\diamond), 6(\bigcirc))$. 